\title{
A Self-Adaptive Upwinding Method for Large Eddy Simulation of Turbulent Flows on Unstructured Elements
}

\author{
Nima Tajallipour ${ }^{1}$, Babak Babaee Owlam ${ }^{2}$, Marius Paraschivoiu ${ }^{3}$ \\ Concordia University, Montreal, Quebec, H3G 1M8, Canada
}

\begin{abstract}
A self-adaptive upwinding method for Large Eddy Simulation (LES) is proposed to reduce the numerical dissipation of a low order numerical scheme on unstructured elements. This method is used to extend an existing Reynolds-averaged Navier-Stokes (RANS) code to an LES code by adjusting the contribution of the upwinding term to the convective flux. This adjustment is essentially controlled by the intensity of the local wiggle and reduces the upwind contribution in Roe MUSCL scheme. First, the stability characteristic of the new scheme is studied, using a channel flow stability test. It is essential to ensure that the proposed scheme is able to adjust upwinding in the presence of very high gradients and prohibits the divergence of the simulation. Second, the decaying isotropic turbulence is simulated in order to study the capability of the new scheme in generating the suitable decaying rate for the total kinetic energy and also its influence over the slope of energy spectrum at different computational times. Finally, the flow separation phenomena over a NACA0025 profile is numerically investigated and results are compared with experimental data.
\end{abstract}

\section{Nomenclature}

$\begin{array}{lll}\bar{f}(x, t) & = & \text { filtered function } \\ \tilde{f}(x, t) & = & \text { Favre-filtered function } \\ f^{\prime \prime}(x, t) & = & \text { fluctuating component of the filtered function } \\ G(x, \Delta) & = & \text { filter function } \\ \Delta & = & \text { filtering size } \\ x_{i} & = & \text { cartesian coordinates }(\mathrm{i}=1,2,3) \\ \bar{\rho} & = & \text { mean density } \\ \tilde{u}_{i} & = & \text { cartesian components of the filtered velocity } \\ \bar{p} & = & \text { mean pressure } \\ \tilde{e} & = & \text { filtered total energy per unit mass } \\ \tilde{T} & = & \text { Favre-filtered static temperature } \\ \rho_{\infty} & = & \text { reference density } \\ U_{\infty} & = & \text { reference velocity } \\ \mathrm{L} & = & \text { reference length scale } \\ a_{\infty} & = & \text { average ambient speed of sound }\end{array}$

${ }^{1}$ Research Assistant, Department of Mechanical and Industrial Engineering, Concordia University, 1455 de Maisonneuve Blvd. W., EV 14-115, Montreal, Quebec, Canada H3G 1M8, Email:n_tajall@encs.concordia.ca

2 Research Assistant, Department of Mechanical and Industrial Engineering, Concordia University, 1455 de Maisonneuve Blvd. W., EV 4-149, Montreal, Quebec, Canada H3G 1M8, Email: bbabaee@ gmail.com

3 Associate Professor, Department of Mechanical and Industrial Engineering, Concordia University, 1455 de Maisonneuve Blvd. W., EV 14-115, Montreal, Quebec, Canada H3G 1M8, Email: paraschi@encs.concordia.ca 


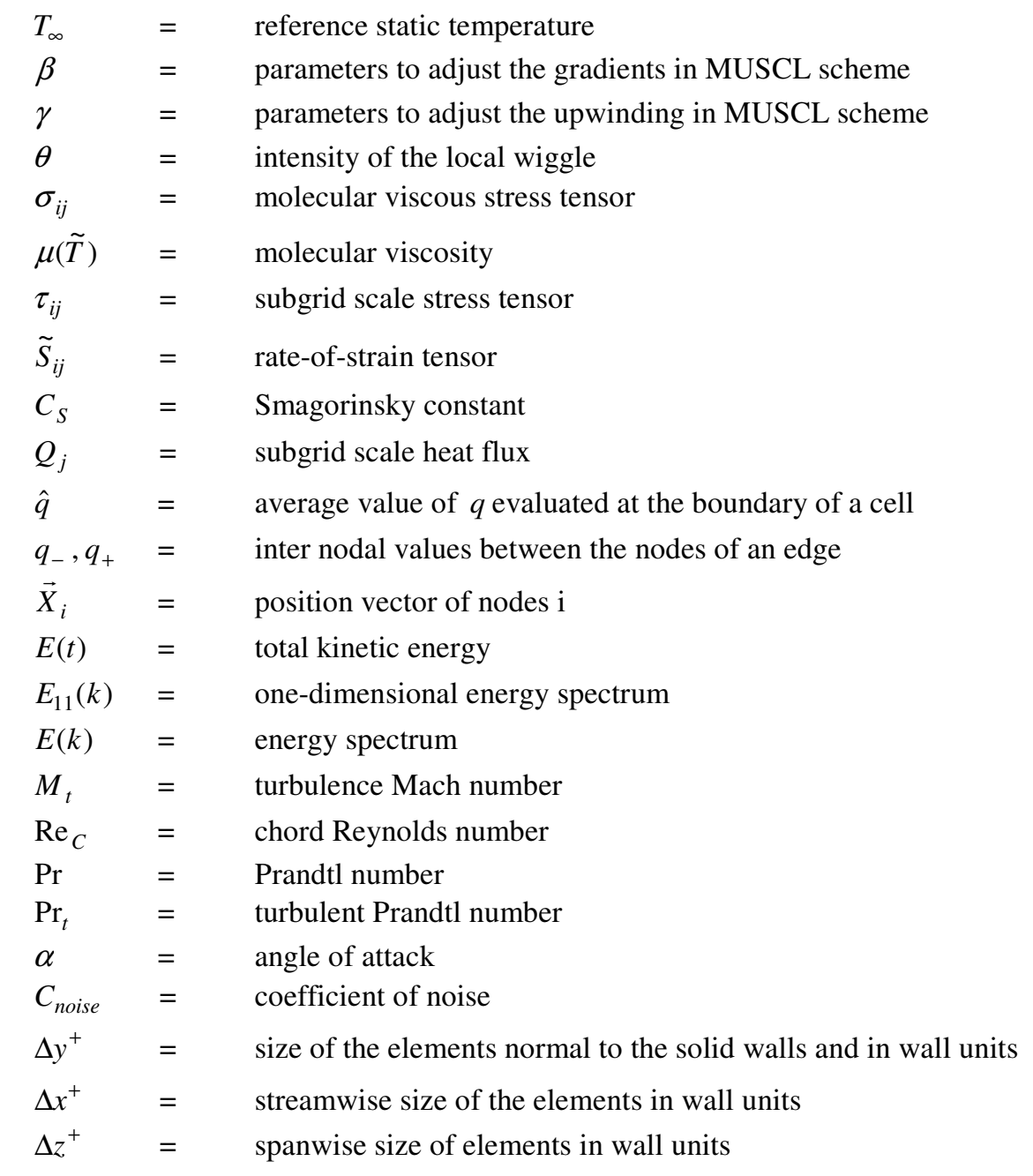

\section{Introduction}

THE importance of applying accurate numerical methods in LES, especially when it involves an upwinding term in the flux calculation has been previously reported in the literature. ${ }^{1-3}$ The upwind schemes, regardless of the applied numerical method, add some artificial dissipation and therefore are known to be much more stable than central difference schemes and are used successfully for RANS simulations. This additional dissipation however can negatively influence the accuracy. Spyropoulos and Blaisdell, ${ }^{1}$ used a $5^{\text {th }}$ order, upwind-biased finite difference scheme for the inviscid flux calculation in order to simulate the turbulent flow inside a channel. They recognized the fact that the upwinding schemes provide artificial dissipation; therefore they have preferred to use the upwind-biased scheme. Mary and Sagaut, ${ }^{2}$ used a $2^{\text {rd }}$ order MUSCL, cell-centered control volume scheme in order to simulate the flow around an airfoil, using structured multi-block meshes. They applied a sensor in order to minimize the 
numerical dissipation originated from its upwinding. Andersson et al., ${ }^{3}$ simulated a compressible jet using a $3^{\text {rd }}$ order upwinding scheme in which the contribution of the upwinding term was decreased to one eighth in order to make the numerical method less dissipative.

The additional dissipation of a numerical method highly depends on the nature of emulated flow and the local resolution of the grid and under some circumstances; it can even be higher than the dissipation of applied subgrid scale model. That is specially the case when a low order numerical discretization as the one applied in this paper, is used. For example, Hahn and Drikakis, ${ }^{4}$ have simulated decaying turbulence and compressible flow around open cavities for low and high order Reynolds numbers. They have concluded that the numerical dissipation of their numerical method was satisfactory and therefore addition of an explicit subgrid model (SGS) was not justifiable.

In this paper, we use the $2^{\text {nd }}$ order Roe-MUSCL flux calculation ( $\beta \gamma$ scheme) for the LES and the objective is to evaluate and possibly control the effects of its numerical dissipative error. In MUSCL ( $\beta \gamma$ scheme), the contribution of the upwinding term is adjusted, using a coefficient $(\gamma)$ which is directly multiplied into that term during the flux calculation. Coefficient $(\beta)$ is also used in order to predict the value of variables at the boundaries of control volume cells.

A complete analysis of the accuracy of MUSCL ( $\beta \gamma$ scheme) and its relation to the structure of generated grid has been reported by Carpentier. ${ }^{5}$ In that study, the dissipative and dispersive error terms of $2 \mathrm{D}$ advection equation have been analyzed using two different meshes (a uniformly distributed triangular mesh and a structured quadrangular mesh). He concluded that the $\beta=1 / 3, \gamma=0.0$ will result in a $4^{\text {th }}$ order dispersive error and a $5^{\text {th }}$ order dissipative error. This high order dissipative error requires the scheme not to have any upwinding $(\gamma=0.0)$. It was also observed that these error terms were functions of the courant number (CFL) related to the applied $4^{\text {th }}$ order Runge-Kutta time discretization scheme. If $\beta=1 / 3$ but $\gamma \neq 0.0$ then the dissipative error will be of $3^{\text {rd }}$ order while the dispersive error will remain of $4^{\text {th }}$ order. In addition, it was also found that the uniform triangular mesh potentially has higher dispersive and dissipative error in comparison to quadrangular mesh.

As a result, there have been several attempts in order to lower the applied value of $\gamma$ and consequently decrease the numerical dissipation of the MUSCL method. For example Bui, ${ }^{6}$ simulated the turbulent flow inside a square duct using Roe MUSCL ( $\beta \gamma$ scheme) and a structured grid. He tried to use the smallest possible value for $\gamma$ for 
which the simulation was still stable. Camarri et al., ${ }^{7}$ applied a second order mixed finite volume-finite element code using MUSCL in order to study the contribution of upwinding to the numerical dissipation and its interaction with SGS. They also used the lowest possible fraction of the upwinding term in order to minimize the dissipative error and also satisfy the stability condition of the simulation. In all of these cases, several simulations were needed to be carried out in order to determine the lowest value of $\gamma$ which was still able to keep the simulation stable.

Ciard et al., ${ }^{8}$ recently developed a new scheme for the unstructured meshes based on finite volumes for inviscid and viscous flux calculations. They adjust the contribution of the upwinding term, using a wiggle detector and therefore there is no need for several simulations to determine the lowest value of $\gamma$. In their method the objective was to completely damp the wiggles of a certain size detected by the sensor. This method however, can partially lead to damping of the energy in the smallest scales captured in the simulation. Since in LES, the smallest scales of simulation fall within the inertial subrange, it is necessary to preserve the energy of such scales. ${ }^{9}$

In this paper, a new self-adaptive upwinding method inspired by Refs. 8 and 10 is developed and implemented. It is compatible with classic numerical schemes of compressible flows based on Roe MUSCL ( $\beta \gamma$ scheme) and is also applicable to unstructured girds. It uses a wiggle detector which has been inspired by Ref. 11. The wiggle detector proposed by Ref. 8 detects the wiggles along three consecutive edges which are not necessarily collinear and it could lead to some uncertainty. Therefore a new method, for wiggle detection is proposed here which is expected to be more reliable. In addition, the developed scheme does not completely damp the wiggles detected by the wiggle detector, but instead permits some wiggles to develop up to a preset threshold of intensity inside the computational domain. The importance of that is showed to be crucial in adjusting the slope of the energy spectrum.

It is generally agreed that turbulent flows are characterized by their unsteadiness and unpredictability and the largest part of the turbulent energy is constructed by truly random motions which could be the remains of old coherent structures. ${ }^{9}$ We also know that in a typical simulation, whenever the grid resolution is not high enough to capture all the scales of the flow (LES), application of a central scheme would generate random fluctuations and wiggles. That is the case when $\gamma$ is lowered by the wiggle detector. As a result, the developed scheme introduces a degree of randomness into the solution which could be favorable and generate more realistic results.

In this paper, first the stability characteristic of the new scheme is studied, using channel flow stability test. It is essential to ensure that the proposed scheme is able to adjust upwinding in the presence of very high gradients and 
prohibits the divergence of the simulation. Second, the decaying isotropic turbulence is simulated in order to study the capability of the new scheme in generating the suitable decaying rate for the total kinetic energy and also its influence over the slope of energy spectrum at different computational times. Finally, the separation of flow over a NACA0025 wing is simulated to further examine the new scheme in a numerically more difficult situation. In the following sections of this paper; the governing equations are introduced, followed by the description of the numerical methodology, then the three numerical tests are presented and the results are compared with experimental data.

\section{Governing Equations}

The governing equations are the spatially filtered compressible Navier-Stokes equations. The spatial filtering separates the small scale eddies of the flow and accumulates their effects in the subgrid scale (SGS) stresses and heat transfer terms. The remaining large energy containing eddies, generated by the large scale turbulent motions and the dynamic motions inside the flow field are simulated.

For an arbitrary function, $\bar{f}\left(x_{i}, t\right)$ is defined as :

$$
\bar{f}\left(x_{i}, t\right)=\int_{D} G\left(x_{i}-\xi_{i}, \Delta\right) f\left(\xi_{i}, t\right) d \xi_{i}
$$

$\Delta$ is the filtering size and is related to the size of the computational mesh. For compressible flows the Favre-filtering operation is defined as:

$$
\tilde{f}\left(x_{i}, t\right)=\frac{\overline{\rho f}}{\bar{\rho}}
$$

By this definition, a variable is decomposed into its Favre-filtered component and fluctuating component according to:

$$
f\left(x_{i}, t\right)=\tilde{f}\left(x_{i}, t\right)+f^{\prime \prime}\left(x_{i}, t\right)
$$

If we assume that the filtering operations commute with the derivative operators and then apply the definitions in Eqs. $(1,2)$ to the compressible Navier-Stokes equations; the filtered governing equations are obtained as follows: ${ }^{12-15}$

$$
\begin{gathered}
\frac{\partial \bar{\rho}}{\partial t}+\frac{\partial \bar{\rho} \tilde{u}_{i}}{\partial x_{i}}=0 \\
\frac{\partial \bar{\rho} \tilde{u}_{i}}{\partial t}+\frac{\partial \bar{\rho} \tilde{u}_{i} \tilde{u}_{j}}{\partial x_{j}}=-\frac{\partial \bar{p}}{\partial x_{i}}+\frac{\partial\left(\tau_{i j}+\tilde{\sigma}_{i j}\right)}{\partial x_{j}}
\end{gathered}
$$




$$
\frac{\partial \bar{\rho} \tilde{e}}{\partial t}+\frac{\partial(\bar{\rho} \tilde{e}+\bar{p}) \tilde{u}_{j}}{\partial x_{j}}=\frac{\partial\left(Q_{j}+\frac{\mu(\tilde{T}) C_{p}}{\operatorname{Pr}} \frac{\partial \tilde{T}}{\partial x_{j}}+\left(\tau_{i j}+\tilde{\sigma}_{i j}\right) \tilde{u}_{i}\right)}{\partial x_{j}}
$$

in which $\operatorname{Pr}=0.715 . \tilde{e}$ is the filtered total energy per unit mass and is defined as:

$$
\bar{\rho} \tilde{e}=\bar{\rho} c_{v} \tilde{T}+\frac{1}{2} \bar{\rho} \tilde{u}_{i} \tilde{u}_{i}
$$

The molecular viscous stress tensor $\sigma_{i j}$ is approximated by:

$$
\tilde{\sigma}_{i j}=\mu(\tilde{T})\left(-\frac{2}{3} \frac{\partial \tilde{u}_{k}}{\partial x_{k}} \delta_{i j}+\frac{\partial \tilde{u}_{i}}{\partial x_{j}}+\frac{\partial \tilde{u}_{j}}{\partial x_{i}}\right)
$$

where $\mu(\tilde{T})$ is molecular viscosity and is calculated by Sutherland equation:

$$
\mu(\tilde{T})=\left(1.711 \times 10^{-5}\right)\left(\frac{\tilde{T}}{273.15}\right)^{2 / 3}\left(\frac{383.55}{\tilde{T}+110.4}\right)
$$

The $\tau_{i j}$ is the subgrid scale stress tensor which is modeled by compressible extension of the Smagorinsky subgrid-scale as follows:

$$
\tau_{i j}=-\left(\overline{\rho u_{i} u_{j}}-\bar{\rho} \tilde{u}_{i} \tilde{u}_{j}\right)=\bar{\rho} C_{S} \Delta^{2} \sqrt{\tilde{S}_{k l} \tilde{S}_{k l}}\left(2 \tilde{S}_{i j}-\frac{2}{3} \tilde{S}_{k k} \delta_{i j}\right)
$$

The rate-of-strain tensor is defined as:

and the model for the filtering size is:

$$
\tilde{S}_{i j}=\frac{1}{2}\left(\frac{\partial \tilde{u}_{i}}{\partial x_{j}}+\frac{\partial \tilde{u}_{j}}{\partial x_{i}}\right)
$$

$$
\Delta=\left(\operatorname{Vol}\left(C_{i}\right)\right)^{1 / 3}
$$

where $\operatorname{Vol}\left(C_{i}\right)$ is the volume of cell $C_{i}$ which belongs to node i. The $Q_{j}$ is the subgrid scale heat flux and is modeled using eddy viscosity model:

$$
Q_{j}=-c_{p}\left(\overline{\rho u_{j} T}-\bar{\rho} \tilde{u} \tilde{T}_{j}\right)=\bar{\rho} C_{P} \frac{C_{S}}{\operatorname{Pr}_{t}} \Delta^{2} \sqrt{\tilde{S}_{k l} \tilde{S}_{k l}} \frac{\partial \tilde{T}}{\partial x_{j}}
$$

in which $\operatorname{Pr}_{t}=0.6$. We simplify the notation by dropping the tidle and overbar signs and also non-dimensionalize the equations using the reference values $\left(\rho_{\infty}, U_{\infty}, \rho_{\infty} U_{\infty}^{2}, L, T_{\infty}\right)$. The governing equations take the following form: 


$$
\begin{aligned}
& \frac{\partial q}{\partial t}+\operatorname{div}\left(\vec{F}^{\text {conv }}+\vec{F}^{\text {diff }}\right)=0 \\
& q=\left\{\begin{array}{c}
\rho \\
\rho u_{1} \\
\rho u_{2} \\
\rho u_{3} \\
\rho e
\end{array}\right\}(16) \\
& F_{j}{ }^{c o n v}=\left\{\begin{array}{c}
\rho u_{j} \\
\rho u_{j} u_{1}+p \delta_{j 1} \\
\rho u_{j} u_{2}+p \delta_{j 2} \\
\rho u_{j} u_{3}+p \delta_{j 3} \\
u_{j}(\rho e+p)
\end{array}\right\} \\
& F_{j}^{d i f f}=\left\{\begin{array}{c}
0 \\
-\sigma_{j 1}-\tau_{j 1} \\
-\sigma_{j 2}-\tau_{j 2} \\
-\sigma_{j 3}-\tau_{j 3} \\
-\frac{\mu(\tilde{T}) C_{p}}{\operatorname{Pr}} \frac{\partial \tilde{T}}{\partial x_{j}}-Q_{j}-u_{k}\left(\sigma_{k j}+\tau_{k j}\right)
\end{array}\right\}
\end{aligned}
$$

\section{Numerical Method}

The numerical method is a mixed finite volume-finite element method, ${ }^{13,16}$ which has been developed to solve the unsteady Navier-Stokes equations. It operates on unstructured grids, using $2^{\text {nd }}$ order MUSCL upwind formulation ( $\beta \gamma$ scheme) for the convective fluxes and a $2^{\text {nd }}$ order finite element method for the diffusive fluxes. As it was mentioned earlier, subgrid scale terms are modeled by the Smagorinsky model. For the decaying isotropic turbulence, we assume $C_{S}=0.01$ but for the flow separation over NACA0025 a much lower value $\left(C_{S}=0.00014\right)$ is used. During the channel flow simulation the subgrid-scale model has been deactivated. A second order implicit scheme is also used for the time discretization. The system of equations is then solved, using an iterative GMRES solver.

\section{A. Self-Adaptive Upwinding Scheme}

Roe-MUSCL method is the base for the convective flux calculation in this work. The normal component of the inviscid flux at the boundaries of neighboring cells is defined as a sum of an average term calculated by fluxes of two nodes belonging to the edge which the flux is calculated along it and an upwinding term.

$$
\vec{F}^{c o n v} \bullet \vec{n}_{i j} \cdot=\frac{1}{2}\left(\vec{F}^{c o n v}\left(q_{+}\right)+\vec{F}^{c o n v}\left(q_{-}\right)\right) \bullet \vec{n}_{i j}-\frac{1}{2}\left|\hat{A}\left(\hat{q}, \vec{n}_{i j}\right)\right|\left(q_{+}-q_{-}\right)
$$

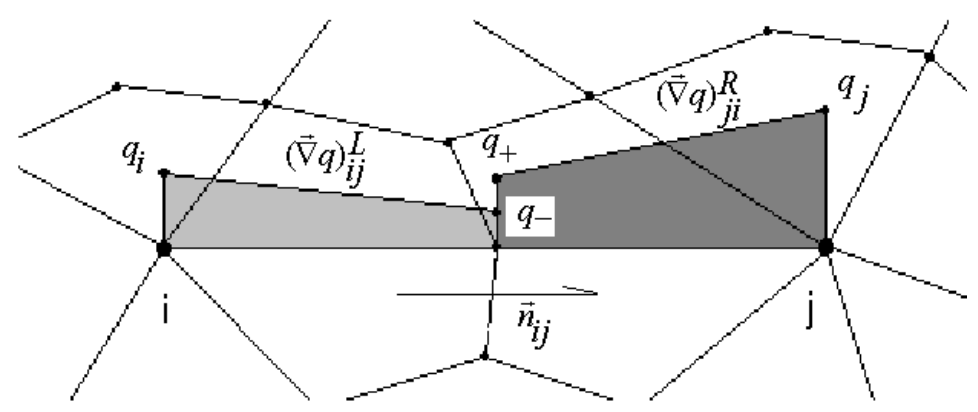

Fig. 1 Control volumes and convective flux calculation. 
$\hat{q}$ is the average value of $q$ evaluated at the boundary of a cell or control volume using Roe method. $q_{-}$and $q_{+}$are calculated by interpolating the numerical flux of those two nodes to the boundaries of the cell $\partial C_{i}$ (inter nodal values between nodes $\mathrm{i}$ and $\mathrm{j})$ :

$$
\begin{aligned}
& q_{-}=q_{i}+\frac{1}{2}\left[(1-\beta)\left(q_{j}-q_{i}\right)+\beta(\vec{\nabla} q)_{i j}^{L} \bullet \vec{n}_{i j}\right] \\
& q_{+}=q_{j}-\frac{1}{2}\left[(1-\beta)\left(q_{j}-q_{i}\right)+\beta(\vec{\nabla} q)_{j i}^{R} \bullet \vec{n}_{i j}\right]
\end{aligned}
$$

This approach is used in order to improve the precision of the method without changing the approximation space. ${ }^{13,16}$ The parameter $\beta$ determines to what extend centeral interpolation is used in order to calculate $q_{+}$and $q_{-}$. As it has been recommended in Ref. 5, we set the value of $\beta$ equal to $1 / 3$ in order to minimize the dissipative and dispersive errors. $(\vec{\nabla} q)_{i j}^{L}$ and $(\vec{\nabla} q)_{j i}^{R}$ are defined as left hand and right hand gradients (Figs. 1,2). These gradients are computed respectively on the upstream $(L)$ and downstream $(R)$ tetrahedrons associated with edge ij (Fig. 2). Local average gradients also can be used in Eqs. $(20,21)$ as an approximation. This is an extension of the MUSCL method to the finite element, because the gradients of the variable vector $(q)$ are computed using the finite element technique.

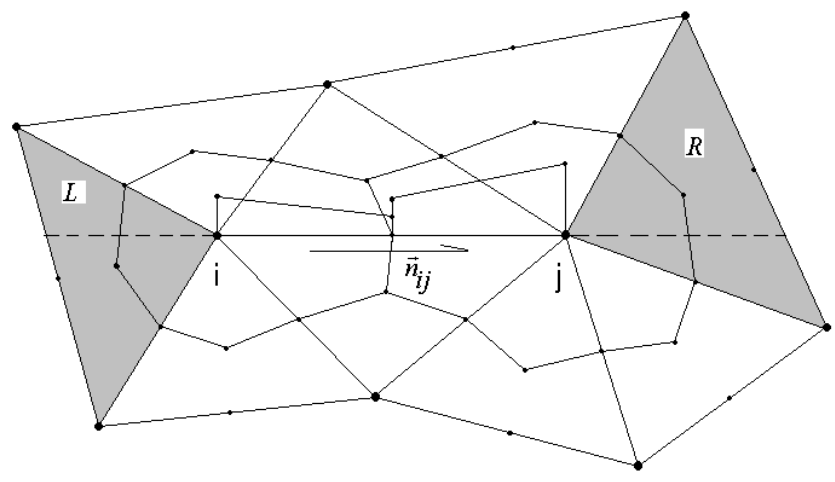

Fig. 2 Convective flux calculation

The Roe-MUSCL has given good results for Euler or laminar simulations but it has been found to be too dissipative for LES. ${ }^{6,10}$ In order to control the amount of Roe upwinding dissipation a coefficient $(\gamma)$ is used, such that:

$$
\vec{F}^{c o n v} \bullet \vec{n}_{i j}=\frac{1}{2}\left(\vec{F}^{c o n v}\left(q_{+}\right)+\vec{F}^{c o n v}\left(q_{-}\right)\right) \bullet \vec{n}_{i j}-\gamma\left\{\frac{1}{2}\left|\hat{A}\left(\hat{q}, \vec{n}_{i j}\right)\right|\left(q_{+}-q_{-}\right)\right\}
$$

where $\gamma$ can range between 0 and 1. In Eq. (22), $\gamma=0$ corresponds to central differencing, and $\gamma=1$ corresponds to the full MUSCL-Roe method. Omitting the Roe upwinding term altogether $(\gamma=0)$ causes all calculations to be 
unstable therefore for a given grid size, a minimum amount of upwinding dissipation is always required in order to provide stability. In general, a finer grid would require a smaller value of $\gamma$.

In order to determine and adjust upwinding parameter $(\gamma)$ dynamically, a wiggle detector has been implemented. It checks to see if the intensity of the local wiggle is higher than a preset value. If this is the case then the scheme increases $\gamma$ linearly towards the full MUSCL-Roe scheme. Otherwise the scheme is more centered and $\gamma$ is decreased.

In the present computations we extend the wiggle definition, given in Ref. 11 to our numerical method. A wiggle is assumed to be present along an arbitrary edge, if the coefficient of direction changes twice along the edge in that direction. That is, if for any flow variable $\Phi(\Phi \in[\rho, u, v, w, p])$

$$
\begin{aligned}
& \left(\Phi_{\mathrm{i}}-\Phi_{\mathrm{i}-1}\right)\left(\Phi_{\mathrm{i}+1}-\Phi_{\mathrm{i}}\right)<0 \\
& \left(\Phi_{\mathrm{i}+2}-\Phi_{\mathrm{i}+1}\right)\left(\Phi_{\mathrm{i}+1}-\Phi_{\mathrm{i}}\right)<0
\end{aligned}
$$

are true, then a wiggle is present. A simplified example is illustrated in Fig. 3. In this example there is a wiggle along the edge connecting nodes $i$ and $i+1$, but there is no wiggle along the edge connecting nodes $i-1$ and $i$.

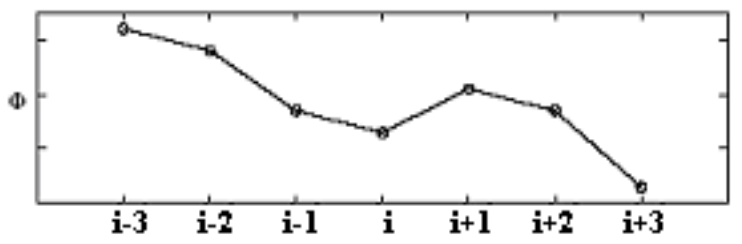

Fig. 3 The definition of a wiggle in the present computations (Ref. 11).

A new method is developed here to be a more general and appropriate approach for the purpose of LES. Let's consider a tetrahedron having ij as an edge (Fig. 2). Along ij we compute $(\vec{\nabla} \Phi)^{C}$ (" $\mathrm{C}$ "' as centered) which $(\vec{\nabla} \Phi)^{C} \bullet \vec{n}_{i j}=\left(\Phi_{j}-\Phi_{i}\right) /\left|\vec{X}_{j}-\vec{X}_{i}\right| . \vec{X}_{i}$ and $\vec{X}_{j}$ are the position vectors of nodes $\mathrm{i}$ and $\mathrm{j}$ respectively. We replace then inequalities $(23,24)$ with the followings:

$$
\begin{aligned}
& {\left[(\vec{\nabla} \Phi)_{i j}^{L} \bullet \vec{n}_{i j}\right]\left[(\vec{\nabla} \Phi)^{C} \bullet \vec{n}_{i j}\right]=\left[(\vec{\nabla} \Phi)_{i j}^{L} \bullet \vec{n}_{i j}\right]\left[\left(\Phi_{j}-\Phi_{i}\right) /\left|\vec{X}_{j}-\vec{X}_{i}\right|\right]<\theta \leq 0} \\
& {\left[(\vec{\nabla} \Phi)_{j i}^{R} \bullet \vec{n}_{i j}\right]\left[(\vec{\nabla} \Phi)^{C} \bullet \vec{n}_{i j}\right]=\left[(\vec{\nabla} \Phi)_{j i}^{R} \bullet \vec{n}_{i j}\right]\left[\left(\Phi_{j}-\Phi_{i}\right) /\left|\vec{X}_{j}-\vec{X}_{i}\right|\right]<\theta \leq 0}
\end{aligned}
$$

If inequalities $(25,26)$ are satisfied then the intensity of the wiggle is more than the preset value $(\theta)$ and $\gamma$ should be increased. This increment is a linear function of $\theta-\operatorname{Min}\left\{\left[(\vec{\nabla} \Phi) \bullet \vec{n}_{i j}\right]\left[(\vec{\nabla} \Phi)^{C} \bullet \vec{n}_{i j}\right]\right\}$. On the other hand, when inequalities $(25,26)$ are not satisfied, it implies that the intensity of the wiggle is less than the preset value $(\theta)$ 
and $\gamma$ can be decreased. This time, the decrement would be a linear function of $\operatorname{Max}\left\{\left[(\vec{\nabla} \Phi) \bullet \vec{n}_{i j}\right]\left[(\vec{\nabla} \Phi)^{C} \bullet \vec{n}_{i j}\right]\right\}-\theta$. In both linear functions, $(\vec{\nabla} \Phi) \in\left[(\vec{\nabla} \Phi)_{i j}^{L},(\vec{\nabla} \Phi)_{j i}^{R}\right], \Phi \in[\rho, u, v, w, p]$ and the value of $(\gamma)$ is chosen between 0 and 1 . The value of $\theta$ is either negative or zero and usually has a small absolute value ( $\approx-0.00001)$. The idea is to use the products of the gradients as a way to measure the intensity of a local wiggle.

For DNS, the grid is fine enough to capture the smallest scales of eddies present in the flow field. Therefore the highest mode of energy spectrum $(E(k))$ of the flow is lower than the highest mode that the numerical method can capture. In other words, the smallest eddies present in the flow have an average diameter which is fairly bigger than local average size of the grid (h) and therefore no energy is expected to be present in the scales belonging to h. It implies that there should not be any local wiggle present in the flow and $\theta$ in the non-equalities $(25,26)$ must be set to zero. For LES, however, the situation is different because even in the best cases, the cutoff mode is expected to fall within inertial subrange and therefore there will be some energy in the highest scales which are expected to be captured by the simulation. Therefore, $\theta$ is replaced by a negative and relatively small value which will represent the existence of energy in the smallest scales and by changing the value of $\theta$, the amount of that energy is adjusted.

\section{Numerical Results}

At first, a channel flow simulation is carried out to test the stability of the scheme against the presence of strong gradients and fluctuations in the flow and to measure the ability of the scheme to adjust itself under such circumstances. Afterwards, the decaying isotropic turbulence is simulated and the influence of the self-adaptive upwinding scheme over the total kinetic energy and the energy spectrum is studied. Its interaction with the external Smagorinsky SGS is also investigated. These test cases are validation and calibration steps for the development of the scheme. Finally, the scheme is used to simulate flow separation over a NACA0025 airfoil and the results are compared with the experimental data.

\section{A. Channel Flow Simulation}

In the Channel flow test, the stability characteristics of the numerical method against strong gradients and fluctuations, artificially generated by a noise term in the flow initialization, is investigated. It is expected that the computed velocities result in a bounded total kinetic energy. If the scheme fails to satisfy this condition, it can be 
concluded with certainty that the numerical method is not suited to model turbulent flows. On the other hand, if it fulfills this condition, it does not mean automatically that it is a good model.

In this test, the flow through a channel presented in Fig. 4 is simulated. A similar test has been presented for an incompressible fluid simulation in Ref. 17, using hexahedral and tetrahedral elements. In the simulation presented here only tetrahedral elements are used. The discretized domain contains 2302 tetrahedrons, 9658 nodes and 2532 boundary faces. A rather coarse mesh is used in this simulation in order to reflect the typical situation in LES of turbulent flows in which there are too few degrees of freedom available for the simulated Reynolds number. The time step $(\Delta t)$ of the simulation is set to 0.01 as it is the case in Ref. 17. At each time step the residual is decreased 6 degrees of magnitude to provide accurate results.

The inflow boundary condition is defined as:

$$
\vec{U}(t, 0, y, z)=\left(\begin{array}{c}
4 y(1-y) \\
0 \\
0
\end{array}\right)
$$

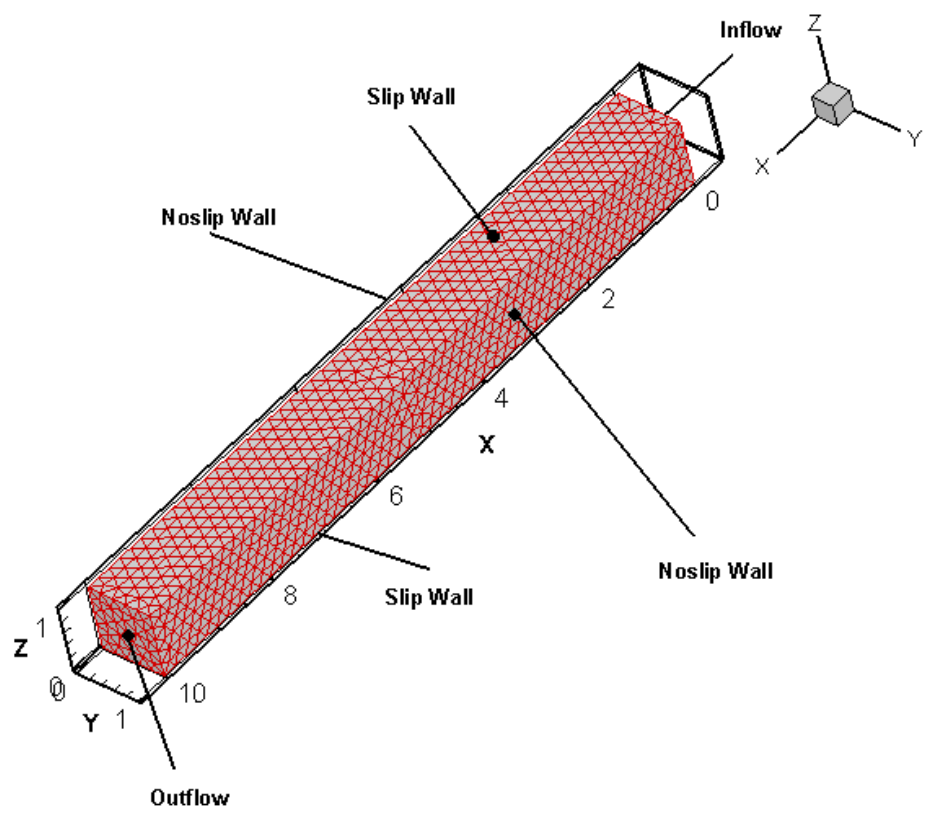

Fig. 4 Channel flow, grid and boundary conditions.

On the lateral walls, a noslip boundary condition is imposed and on the top and bottom walls a free slip boundary condition is applied (Fig. 4). The flow leaves the channel at the outflow boundary condition and pressure is kept fixed over that face. The initial velocity is given by: 


$$
\vec{U}(0, \vec{X})=\left(\begin{array}{c}
4 y(1-y) \\
0 \\
0
\end{array}\right)+C_{\text {noise }}\left(\begin{array}{c}
-4 \pi \sin (4 \pi y) \\
-3 \pi \sin (3 \pi z) \\
3 \pi \cos (3 \pi x)
\end{array}\right)
$$

Without presence of any noise in the initial condition $\left(C_{\text {noise }}=0.0\right)$

$$
\begin{gathered}
\vec{U}(t, x, y, z)=\left(\begin{array}{c}
4 y(1-y) \\
0 \\
0
\end{array}\right) \\
P(t, x, y, z)=-8 v(x-10)+P_{0}
\end{gathered}
$$

is a solution of the Navier-Stokes equations (Eqs. 4-6). The total kinetic energy of the flow inside the channel (steady condition) is estimated to be 2.666 by integrating the velocity distribution given by the Eq. (29) over the entire nondimensionalized domain.

The nondimensional molecular viscosity is assumed to be $v=10^{-5}$ and the Reynolds number of the flow, based on the average inflow and the height of the channel $\mathrm{L}$ is:

$$
\operatorname{Re}=\frac{\bar{U} L}{v}=\frac{2}{3} 10^{5} \approx 66667
$$

In this simulation the Mach number is set to the value of 0.01 in order to keep flow regime close to incompressible. The total kinetic energy of the computational domain is computed by:

$$
E_{\text {total kinetic energy }}=\sum_{n=1}^{N}\left(\left(\sum_{i=1}^{3} \frac{u_{i}^{2}(n)}{2}\right) \times \operatorname{Vol}\left(C_{n}\right)\right)
$$

where $\mathrm{N}$ is defined as total number of nodes inside the domain and $\operatorname{Vol}\left(C_{n}\right)$ is the volume of cell $C_{n}$ which belongs to node $\mathrm{n}$.

In the channel flow simulation, $\gamma$ is assumed to be chosen within a range with fixed limits for each simulation. The limits of that range fall within $[0,1]$. This makes it possible to adjust the average effect of upwinding in every test case. To test the stability of the self-adaptive scheme, the permitted intensity of the local wiggles $(\theta)$ is set to zero, intended to completely damp the local wiggles. Considering that the grid resolution is very low and $\theta$ has been set to zero, we expect a laminar solution despite the fact that the Reynolds number is relatively high.

The different computations, as summarized in Table 1, were performed in order to evaluate the stability of the scheme against strong fluctuations which might be present in every typical LES. 
Table 1 Explanation of different cases for channel flow simulation

\begin{tabular}{|c|c|c|c|c|c|c|c|}
\hline \multirow{2}{*}{$\begin{array}{c}\text { Case } \\
\# \\
\end{array}$} & \multirow{2}{*}{$\theta$} & \multicolumn{2}{|c|}{ Upwinding Parameters } & \multirow{2}{*}{$C_{\text {noise }}$} & \multirow{2}{*}{$C_{S}[$ Smag. $]$} & \multicolumn{2}{|c|}{$E_{\text {Kinetic }}$} \\
\hline & & Up & Low & & & $t=0$ & $\mathrm{t}=10$ \\
\hline 1 & 0.0 & 1.0 & 1.0 & 0.0 & 0.0 & 2.6617 & 2.3296 \\
\hline 2 & 0.0 & 1.0 & 0.43 & 0.0 & 0.0 & 2.6617 & 2.6508 \\
\hline 3 & 0.0 & 1.0 & 0.1 & 0.0 & 0.0 & 2.6617 & 2.9446 \\
\hline 4 & 0.0 & 1.0 & 0.0 & 0.0 & 0.0 & 2.6617 & 3.0023 \\
\hline 5 & 0.0 & 1.0 & 0.43 & 0.01 & 0.0 & 2.73813 & 2.6510 \\
\hline 6 & 0.0 & 1.0 & 0.43 & 0.1 & 0.0 & 10.2229 & 2.6738 \\
\hline
\end{tabular}

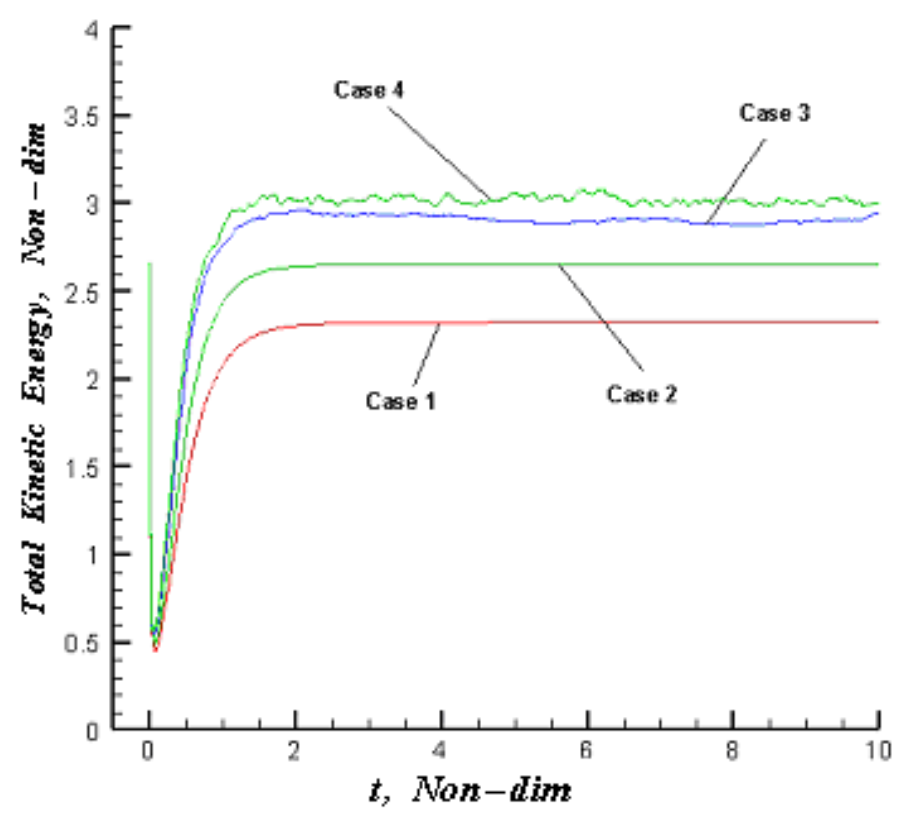

Fig. 5 Channel flow simulation (Cases 1, 2, 3, 4).

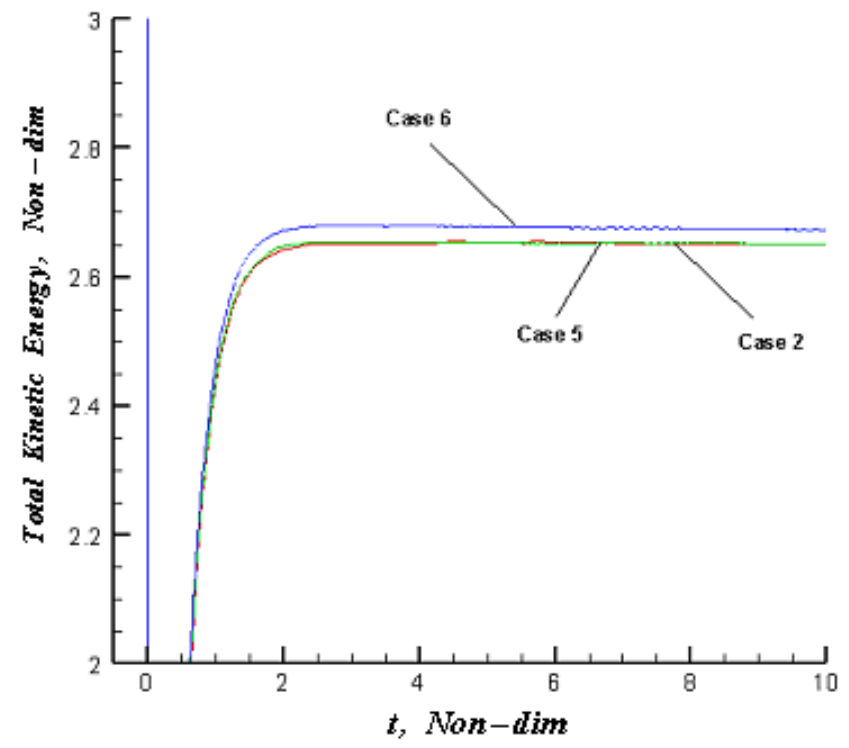

Fig. 6 Channel flow simulation (Cases 2, 5, 6). 
When full upwinding (case 1) is considered, the total kinetic energy of the system is somewhat lower that the expected value of 2.666. Recall that the flow is not resolved well enough by the mesh and therefore Eq. (32) is not a good approximation for the integration operation used to calculate the estimated total kinetic energy. In cases 2, 3, and 4, the lower limit of upwinding parameter $(\gamma)$ is decreased (Table 1) and it reduces the average effects of upwinding respectively. By decreasing $\gamma$ the flux calculation scheme becomes more central which makes the simulation more unstable and therefore the wiggles gradually start to appear inside the domain. The self-adaptive scheme tries to locally adjust $\gamma$ in order to damp those wiggles and as a result the simulation still remains stable. However, a gradual increase in the total kinetic energy is observed as it is illustrated in Fig. 5. In case 4 the effects of those random wiggles appear even further and the total kinetic energy is showing chaotic fluctuations. That is an indication of a fairly unstable flow regime present inside the channel even though the total kinetic energy has still remained bounded. In case 2, the total kinetic energy becomes very close to 2.666 and therefore for the next part of this test, the lower limit of upwinding term is kept equal to 0.43. In Fig. 6 the effect of initial noise is investigated using cases 2,5 and 6 . As it is seen in the graph, the self-adaptive upwinding method is showing a very good stability characteristic despite the fact that the value of 0.1 for noise coefficient is ten times higher than the value used in Ref. 17.

\section{B. Decaying Isotropic Turbulence}

The simplest kind of turbulence is isotropic and, therefore, isotropic turbulence forms a natural starting point for the study and simulation of turbulence. This flow has been considered in many earlier and recent investigations. ${ }^{18-23}$ The experiment of the decay of isotropic turbulence by Comte-Bellot and Corrsin (CBC) ${ }^{24}$ is used to validate our simulation. In the experiment, turbulence was generated using a biplane, square rod grid with mesh size $M=5.08 \mathrm{~cm}$ which was placed inside a flow with the uniform velocity of $U_{0}=10 \mathrm{~m} / \mathrm{s}$. The Reynolds number based on the grid spacing is $\operatorname{Re}_{M}=U_{0} M / v=34000$. The measurements were performed downstream of the grid at three consecutive stations $\left(U_{0} t_{C B C} / M=42,98\right.$ and 171): The dimensional time in the experiment $\left(t_{C B C}\right)$ is defined as:

$$
t_{C B C}=\int_{0}^{x} \frac{d x}{U(x)}
$$


in which $\mathrm{x}$ is the downstream distance from the grid and $\mathrm{U}(\mathrm{x})$ is the mean velocity. Because the same flow passes through all the stations we can use the measured data to verify the validity of the numerical results at three different computational times. The simulation is carried out inside a box with a size larger than the integral length scale and much smaller than the wind tunnel's cross section. 32768 nodes and 178746 tetrahedrons are used in this model. Each edge of the box has been divided by 32 nodes into segments of equal size and then all elements are distributed uniformly inside the domain. Periodic boundary condition is imposed on all sides of the box.

In Ref. 24 one-dimensional energy spectrum $E_{11}$ at $U_{0} t_{C B C} / M=42$ is reported which can be approximated by a logarithmic polynomial as bellow: ${ }^{18,25}$

$$
\log _{e} E_{11}(k)=A_{0}+A_{1} \log _{e} k+A_{2}\left(\log _{e} k\right)^{2}+A_{3}\left(\log _{e} k\right)^{3}+A_{4}\left(\log _{e} k\right)^{4}
$$

In the isotropic turbulence, the energy spectrum $E(k)$ can be obtained from $E_{11}(k)$ by:

$$
E(k)=\frac{1}{2} k^{3} \frac{\partial}{\partial k}\left(\frac{1}{k} \frac{\partial E_{11}}{\partial k}\right)
$$

This yields to:

$$
\begin{aligned}
E(k)= & E_{11}\left\{\frac{1}{2}\left[A_{1}+2 A_{2} \log _{e} k+3 A_{3}\left(\log _{e} k\right)^{2}+4 A_{4}\left(\log _{e} k\right)^{3}\right]^{2}\right. \\
& \left.+A_{2}-A_{1}+\left(3 A_{3}-2 A_{2}\right) \log _{e} k+\left(6 A_{4}-3 A_{3}\right)\left(\log _{e} k\right)^{2}-4 A_{4}\left(\log _{e} k\right)^{3}\right\}
\end{aligned}
$$

in which coefficients are reported in Table 2.

Table 2 Coefficient of energy spectrum at $U_{0} t_{C B C} / M=42$

\begin{tabular}{cc}
\hline \hline Coefficient & Value \\
\hline$A_{0}$ & 4.7935398 \\
$A_{1}$ & -1.3284141 \\
$A_{2}$ & -0.2146974 \\
$A_{3}$ & -0.0314604 \\
$A_{4}$ & -0.0169870 \\
\hline \hline
\end{tabular}

The initial velocity field for the simulation is created by superimposing Fourier modes having prescribed energy spectrum as Eq. (36) but random phases (Fig. 7). The method has been described in Ref. 18 and 25. The initial pressure distribution is obtained from the incompressible Poisson equation using the initial velocity field. A slight difference is present between $E(k)$ reported by $\mathrm{CBC}$ data ${ }^{24}$ and the applied initialization for $E(k)$ as it is seen in Fig. 7. It is necessary to mention that during the CBC experiment, one-dimensional energy spectrums $\left(E_{11}(k)\right)$ were 
measured at three different locations and then the affiliated energy spectrums $(E(k))$ were calculated using "graphical differentiation of faired curves". It seems that using polynomial curve fitting, which is implemented here, is more accurate than that $\mathrm{CBC}$ method. That is however the case only for $U_{0} t_{C B C} / M=42$. Our attempts to use the polynomial procedure to extract $E(k)$ from $E_{11}(k)$ for $U_{0} t_{C B C} / M=98$ and $U_{0} t_{C B C} / M=171$ did not achieve any better result in comparison to $\mathrm{CBC}$. Therefore, we decided to initialize the simulation using Eq. (36) whereas for comparing the energy spectrum of different simulations at $U_{0} t_{C B C} / M=98$ and $U_{0} t_{C B C} / M=171$ with experiment, the curves provided by $\mathrm{CBC}$ are used.

As mentioned earlier, the decaying turbulence is simulated by considering the fluid to be inside a cube. Each side of this cube has the length of $L_{C}$ which is assumed to be equal to $43.787 \mathrm{~cm}$. This length represents the zerointercept of $E(k)$ in Eq. (36).

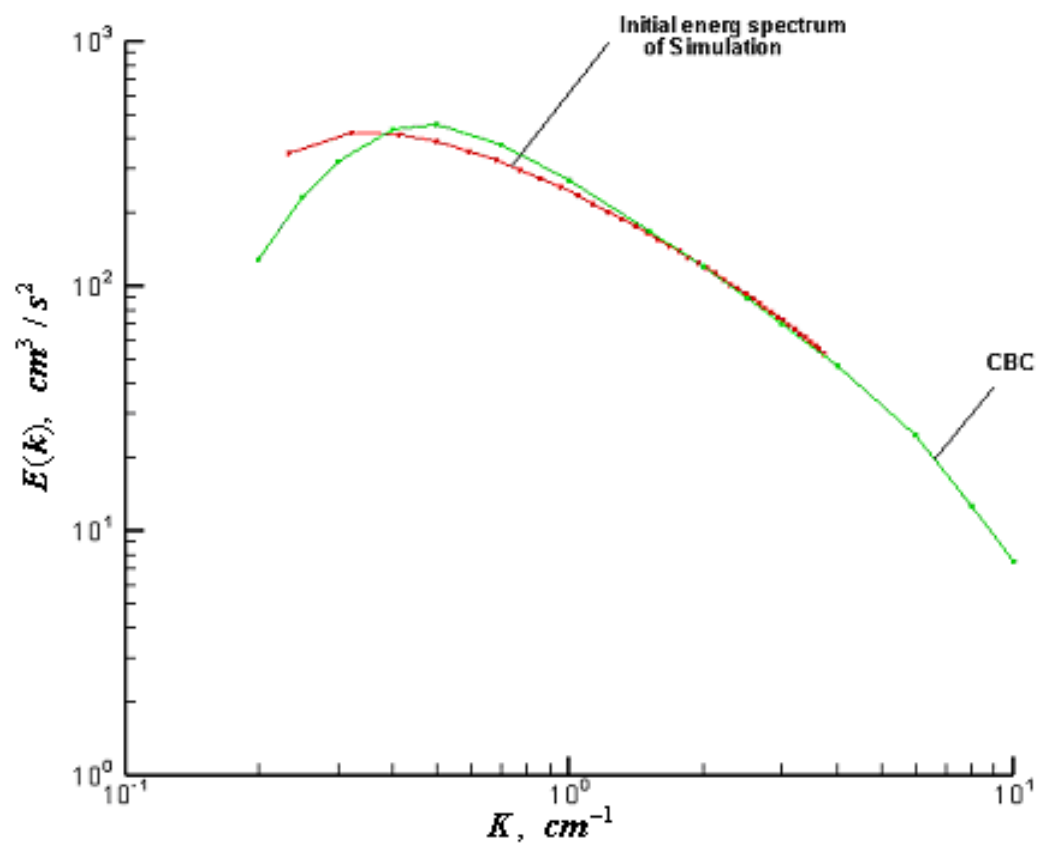

Fig. 7 Energy spectrums at $U_{0} t_{C B C} / M=42$.

The length scale $L_{\infty}=6.96889 \mathrm{~cm}$ and $U_{\infty}=3420 \mathrm{~cm} / \mathrm{s}$ are chosen for nondimensionalization of the governing Eqs.

(4-6) therefore the dimensionless length of cube's edges becomes equal to $2 \pi .^{18} E(k)$ and $k$ have been nondimensionalized by $U_{\infty}^{2} L_{\infty}$ and $L_{\infty}^{-1}$ respectively. The dimensionless time of the simulation (t) is related to $t_{C B C}$ by:

$$
t=\left(\frac{U_{0} t_{C B C}}{M}-42\right) \frac{M}{U_{0}} \frac{U_{\infty}}{L_{\infty}}
$$


and thus

$$
t=2.493\left(\frac{U_{0} t_{C B C}}{M}-42\right)
$$

The flow in the experiment is essentially incompressible. The turbulence Mach number $\left(M_{t}\right)$ of the initialization is equal to $0.98959541 \times 10^{-3}$ where

$$
M_{t}=\sqrt{\overline{u_{i} u_{i}}} / a_{\infty}
$$

and $a_{\infty}=3420 \mathrm{~cm} / \mathrm{s}$.

In earlier works, spectral methods have been mainly used for LES of decaying turbulence. ${ }^{19,21,22,25,26}$ The numerical dissipation was relatively negligible and therefore the SGS model has been mainly the factor to determine the overall dissipation of the simulation. Other numerical methods, however, usually are not as accurate as spectral methods and therefore it is not possible to use the overall judgment to estimate the relative importance of the numerical dissipation. There have been cases, reported in the literature, in which the numerical dissipation of the method was found to be fairly significant. For example, in Ref. 27 compressible isotropic turbulence at zero molecular viscosity with a wide "set of schemes, such as: the Jameson scheme, TVD-MUSCL scheme ( $3^{\text {rd }}$ order) and three schemes within the ENO family (ENO, WENO, MENO)" have been studied. They concluded that "numerical dissipation affects not only the small scales but also the large ones". MUSCL also has been "found to be too dissipative at $64^{3}$ ". Another example would be Ref. 8 , in which numerical method is based on a finite-volume discretization using MUSCL solver. A "self-adaptive method" has been implemented to remedy the over dissipative nature of MUSCL method and has given acceptable results for $32^{3}$. They also reported that "the original scheme was over dissipative" and "for $32^{3}$ grid, the numerical dissipation was in general dominant over the sub-grid scale component".

Table 3 Explanation of different cases for decaying turbulence simulation

\begin{tabular}{ccccc}
\hline \hline \multirow{2}{*}{$\begin{array}{c}\text { Case } \\
\#\end{array}$} & \multicolumn{2}{c}{ Upwinding Parameters } & & \multirow{2}{*}{$C_{S}[$ Smag.] } \\
\cline { 2 - 3 } & Upper Value & Lower Value & $\theta$ & \\
\hline 1 & 1.0 & 1.0 & - & 0.01 \\
2 & 1.0 & 0.0 & 0.0 & 0.01 \\
3 & 1.0 & 0.0 & -0.00001 & 0.01 \\
4 & 1.0 & 0.0 & -0.0001 & 0.01 \\
5 & 1.0 & 0.0 & -0.0001 & 0.1 \\
6 & 1.0 & 0.0 & -0.0001 & 1.0 \\
\hline \hline
\end{tabular}




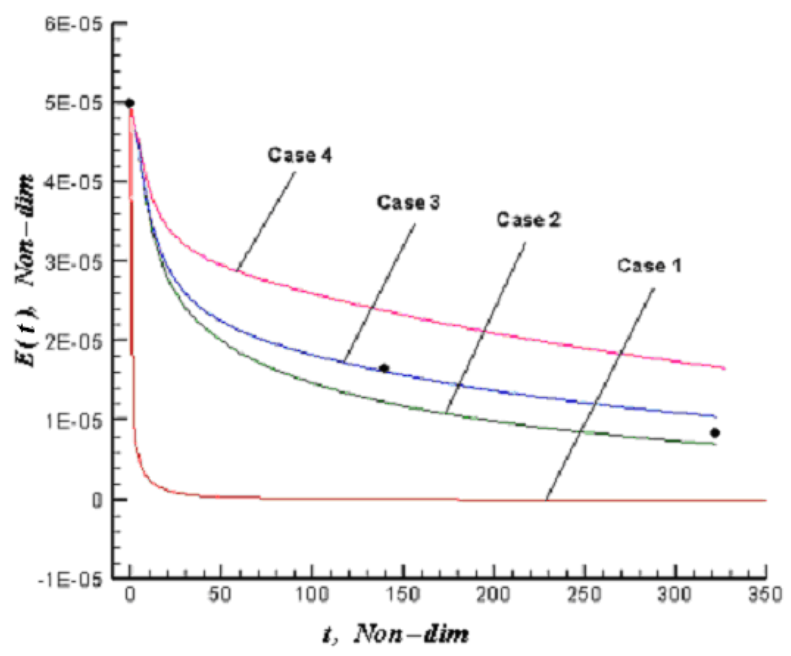

Fig. 8 Total kinetic energy (Cases 1, 2, 3, 4).

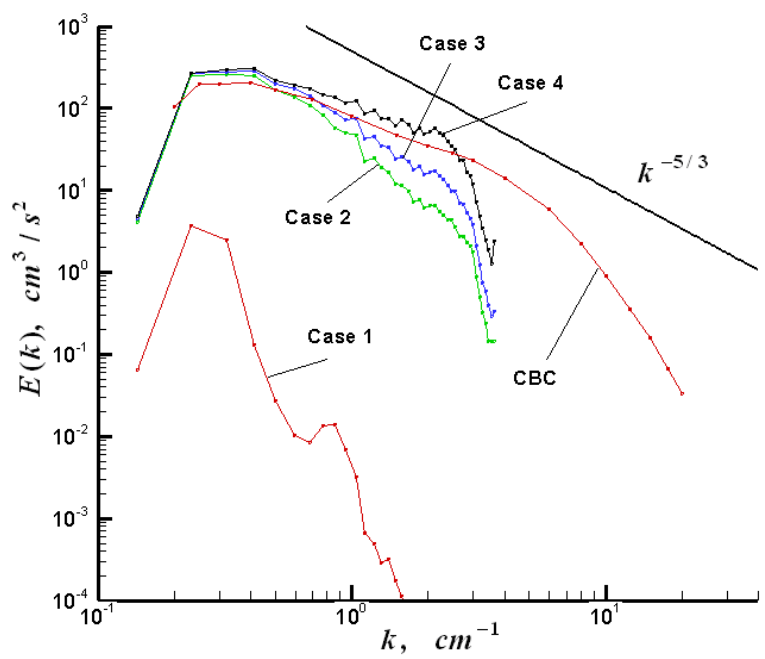

Fig. $9 \mathrm{E}(\mathrm{k})$ at $\boldsymbol{U}_{0} \boldsymbol{t}_{\boldsymbol{C} B C} / \boldsymbol{M}=98($ Cases $\mathbf{1 ,}, \mathbf{2}, \mathbf{3}, \mathbf{4})$.

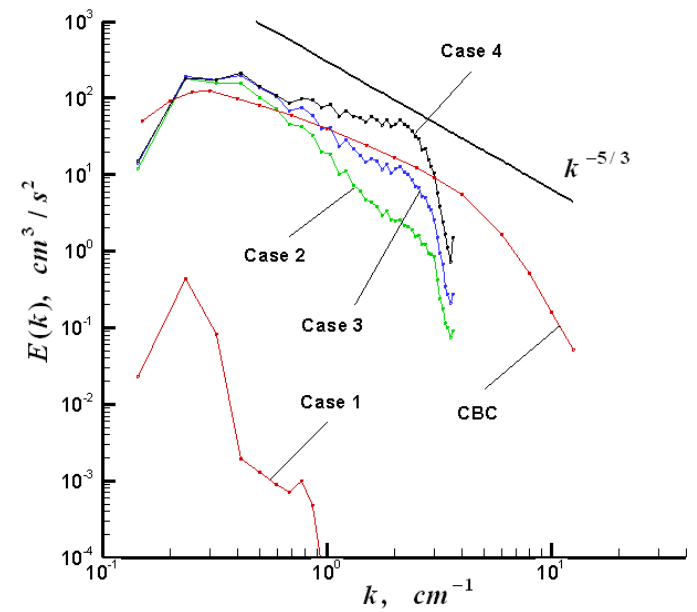

Fig. $10 \mathrm{E}(\mathrm{k})$ at $U_{0} t_{C B C} / M=171($ Cases 1, 2, 3, 4). 


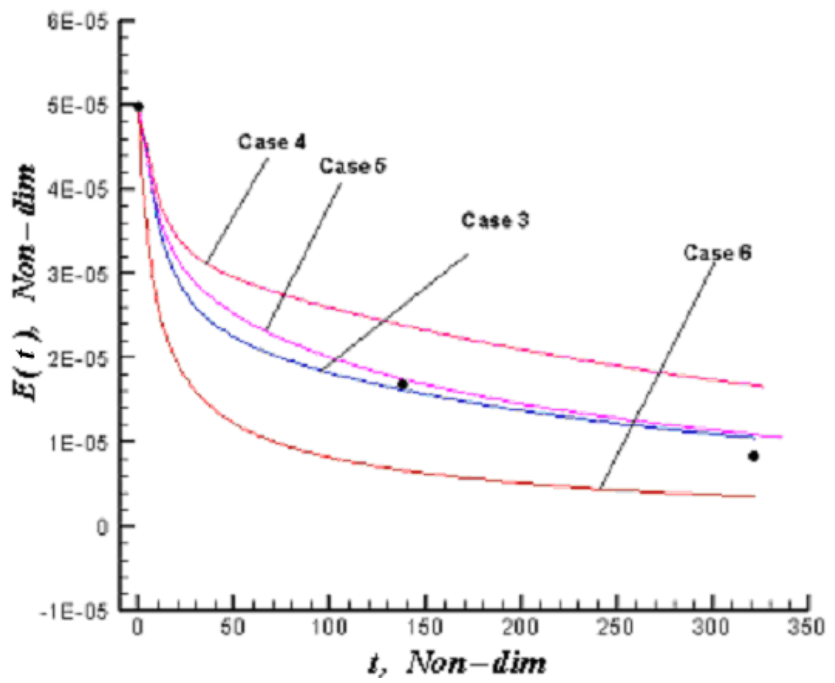

Fig. 11 Total kinetic energy (Cases 3, 4, 5, 6).

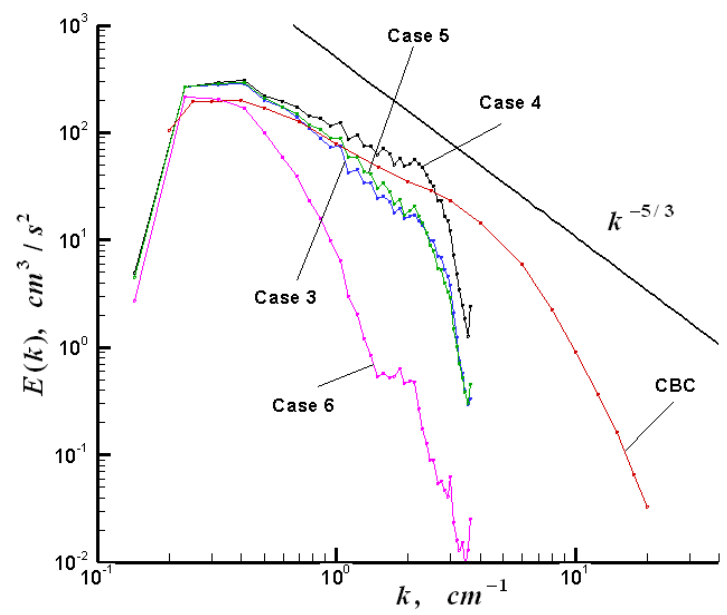

Fig. $12 \mathrm{E}(\mathrm{k})$ at $\boldsymbol{U}_{0} \boldsymbol{t}_{C B C} / \boldsymbol{M}=98($ Cases $\mathbf{3}, \mathbf{4 , 5}, \mathbf{6})$.

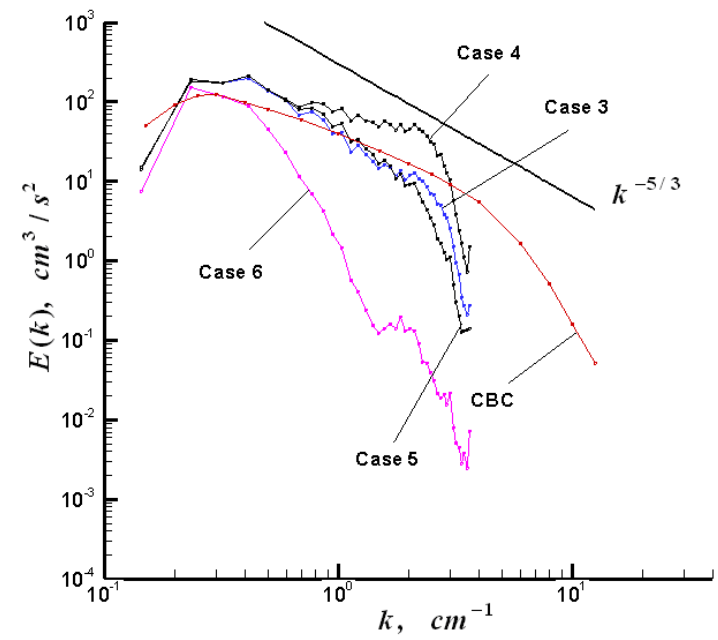

Fig. $13 \mathrm{E}(\mathbf{k})$ at $\boldsymbol{U}_{0} t_{C B C} / \boldsymbol{M}=171($ Cases 3, 4, 5, 6). 
Considering the given discussion, one of the main concerns in this work is to study the effects of numerical dissipation of Roe-MUSCL scheme and introduce a method (self-adaptive upwinding) in order to control its undesirable influence. To study the effects of the self-adaptive upwinding, test cases described in Table 3 are considered. Case 1 shows the set of conditions representing full upwinding. The numerical dissipation is found to be very high (Fig. 8) and therefore it is necessary to significantly decrease the upwinding effect.

In cases 2, 3 and 4 the effect of self-adaptive upwinding is demonstrated. In case 2, self-adaptive upwinding flag is activated and therefore the $\mathrm{E}(\mathrm{t})$ graph is significantly closer to $\mathrm{CBC}$ data points (Fig. 8). In this case $\theta$ is set to 0.0 which means that the self-adaptive scheme tries to dissipate the wiggles in low length scales (Fig. 9). This of course contradicts somehow in principle with the idea of LES in which energy stored at higher modes is expected to play a role in the simulation. Therefore in the next test cases, $\theta$ is decreased, hoping that it will improve the energy distribution in the highest modes. In case $3, \theta$ is decreased to -0.00001 and therefore total kinetic energy becomes a very good match of the experimental data. Results of Case 4, however show that the $\mathrm{E}(\mathrm{t})$ graph can even elevate further upward by decreasing the $\theta$ further. In this case the slope of the energy spectrum is a better match for the CBC data even though the total kinetic energy is clearly higher than the experimental predictions.

As it is seen in Figs. $(9,10)$ the energy is cascaded from lower modes toward higher modes. The energy spectrum is showing a dissipative nature at higher modes while it is not enough dissipative in relatively low modes and an undesired overshoot is present. By decreasing $\theta$, not only the energy in higher modes is increase, but also some energy in lower modes is accumulated therefore it is difficult to achieve a complete match between numerical and experimental data as it was seen in the case of spectral methods.

In case 4, 5 and 6 the effect of explicit SGS is studied (Figs. 11,12,13). By increasing the Smagorinsky constant the energy in the domain is more dissipated and that results in different levels of $E(t)$ as seen in Fig. 11.We conclude that the self-adaptive upwinding scheme has significantly improved the results as it was explained above. It was also found that the overall dissipative nature of simulation is affected by both SGS model and the numerical method.

\section{Flow Separation Detection over NACA0025}

In this section, we want to investigate the performance of the self-adaptive scheme for the simulation of flow separation over an airfoil which is a more challenging problem. The experimental data of Yarusevych ${ }^{28}$ is used to validate the simulation of the flow around a NACA0025 profile. Yarusevych ${ }^{28}$ investigated the separation 
phenomena on the upper surface of the NACA0025 airfoil at Reynolds number equal to 100000. The experiment was conducted in a low-turbulence recirculating wind tunnel at the University of Toronto. The wind tunnel has 5-mlong octagonal test section with a span of $0.91 \mathrm{~m}$, and height of $1.215 \mathrm{~m}$. The airfoil is mounted horizontally inside the wind tunnel, $0.4 \mathrm{~m}$ downstream of the contraction section. The airfoil's surface is equipped with 65 pressure taps to measure the pressure distribution along the upper and lower surfaces in the mid-span. Further details about the experiment can be found in Ref. 28 and 29.

In our simulation, the objective is to verify the ability of the proposed scheme to predict the location of the separation point and also to capture the recirculation zone. The simulation is carried out in a flow with the chord Reynolds number $\left(\operatorname{Re}_{C}\right)$ of 100000 and at the angles of attack equal to 0 and 5 degrees. The chord length is considered to be $0.3 \mathrm{~m}$ as in the experiment. The span length is chosen one chord in order to avoid the walls effects at mid-span although it is $0.15 \times$ chord in the experiment. The computational domain has $0.5 \times$ chord upstream of the leading edge and one chord downstream of the trailing edge. The height of the test section is $4 \times$ chord equal to the height of the wind tunnel.

To accurately capture the separation phenomena over the upper surface of the wing, a very fine boundary layer mesh has been generated. It is compromised of 50 layers of structured mesh with the ratio of 1.05 which smoothly mix with the grids outside of boundary layer. Mesh resolution for LES in the regions near to the solid walls is usually assumed to be in the order of unity in the normal direction $\left(\Delta y^{+} \approx 1\right)$, in order to accurately resolve the velocity gradients. ${ }^{30}$ In this simulation, two models with different mesh resolutions are generated. The model with the finer mesh has the resolution of $\Delta y^{+} \approx 1$ and $\Delta x^{+}=\Delta z^{+} \approx 20$ (streamwise and spanwise spacing) at the boundary layer regions, whereas the coarser mesh has the minimum grid size of $\Delta y^{+} \approx 10$. As it will be shown later, the coarse mesh does not capture the separation phenomena at all. The largest edges inside both grids have the length equal to $\Delta x^{+}=\Delta y^{+}=\Delta z^{+} \approx 100$ in the far field regions. This helps to have the required resolution near the solid walls. The fine mesh is composed of about 3 million nodes and 9.5 millions elements and is decomposed into 64 sub-domains in order to be run in parallel.

As for the boundary conditions, the velocities and density are imposed at the inflow plane. There are four variables which are assigned according to farfield properties and one comes from the numerical solution at the inlet. At the outflow plane, static pressure is fixed, therefore one variable is set according to the physical domain and the 
remaining four variables are calculated from the numerical solution. For this simulation, the streamwise velocity distribution is set to $u=U_{\infty} \cos \alpha$ while the velocity distribution in the two spanwise directions is set to $v=0$ and $w=U_{\infty} \sin \alpha$ respectively. The free stream velocity is shown by $U_{\infty}$ and $\alpha$ represents the angle of attack. On the solid walls (airfoil surface and channel walls), zero velocity condition is applied in all directions to the surface. In addition, to avoid the influence of heat transfer through the walls, the adiabatic boundary condition is applied to the solid walls.

In the simulation, the time step is controlled by the CFL number which linearly increase from 1 to 5 , therefore the maximum CFL is limited to 5 and the maximum time step is set to be $\Delta \mathrm{t}=0.0001 \mathrm{sec}$. The simulation continues running until the flow becomes statistically steady. The best way to make sure that the flow is fully developed and steady is to examine the time history of the residuals and the forces on the airfoil. Afterwards, the sampling data statistics are extracted. The simulation is continued for a period of time and the quantities are averaged. This period is much longer than the period of flow oscillations.

According to the results, when the angle of attack is equal to 5 degrees and the self-adaptive upwinding method is not active (full upwinding), separation is not captured and all the streamlines in the flow field follow the airfoil's surface. When the self-adaptive upwinding is activated, the flow separation occurs on the upper surface of the airfoil and the recirculation zone is generated; however it fails to reattach. As it can be seen, the flow separates on the upper surface of the airfoil and fails to reattach. The separation point is the specific location over the wing where the boundary layer tends to separate from the solid body as a result of the local adverse pressure gradient.

In Fig. 14, the pressure coefficient $\left(C_{P}\right)$ is plotted against the distance from the leading edge of the airfoil at Reynolds number of 100000 and $\alpha=5^{\circ}$. As it is seen, the original scheme (with full upwinding) is not able to capture the separation and the flow remains attached to the wing. The self-adaptive upwinding method shows its positive influence by decreasing the numerical dissipation and allowing the separation to take place. The location of separation for the angle of attack equal to 5 degrees, is estimated to be around $\mathrm{x} / \mathrm{chord}=0.31$ while the experimental data reports $\mathrm{x} / \mathrm{chord}=0.30$ for the separation location at the same angle of attack. As it can be seen in this graph, the region downstream of the separation point has almost constant pressure. Since the boundary layer does not reattach to the airfoil's surface downstream of the separation point, constant pressure region extends up to the trailing edge. The separation point however moves towards the trailing edge as the angle of attack is decreased. The pressure coefficient over the lower surface of the wing is shown in Fig. 15. 
In Fig. 16, the pressure coefficient is plotted for $\alpha=0^{\circ}$. As expected due to the symmetrical profile of the airfoil and zero incidence angle of attack the upper and lower pressure distributions overlap. The boundary layer separates at approximately $\mathrm{x} / \mathrm{chord}=0.48$ while the experiment reported it to happen at $\mathrm{x} / \mathrm{chord}=0.37$.

The ability of capturing the separation phenomena seems to have direct relation to mesh resolution as well. In Fig. 17, the pressure coefficient for the course mesh over the upper and lower surfaces using self-adaptive upwinding scheme is reported. As it is seen, the simulation on the course mesh is not able to capture the separation.

One of the features of LES in respect to RANS is its capability to capture unsteady motions. The unsteady development of the separated shear layer and the periodic vortex shedding are considered among important features of this simulation. ${ }^{31}$ Therefore, for better understanding of the unsteady flow in the separated region, instantaneous streamlines and velocity contours for different simulation times are shown for one shedding cycle in Figs. (18-22). The time step is fixed at $0.0001 \mathrm{~s}$. Since the flow is unsteady, the streamlines are changing with time and the vortex shedding can be observed at the trailing edge region. As can be seen in these figures, the cycle starts in Fig. 18 at 1.0s and terminates in Fig. 22 at 1.067s. This shows that a complete vortex shedding period takes almost 0.067 seconds based on our simulation.

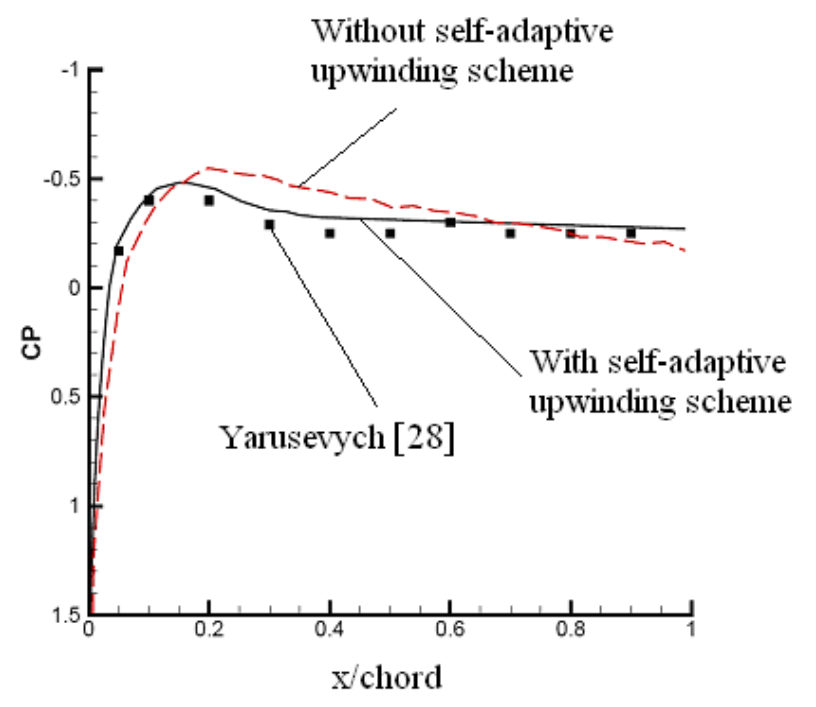

Fig. 14 Pressure coefficient over the upper surface ( $\alpha=5^{\circ}$, fine mesh).

To highlight the enhanced capabilities of the code presented here, the same flow conditions are simulated using a standard CFD commercial code. FLUENT is chosen as the CFD tool because its LES capability has been upgraded in version 6.2 with several enhancements in SGS modeling and also in its numerics, such as Bounded Central Differencing (BCD) and non iterative time advancement (NITA). 


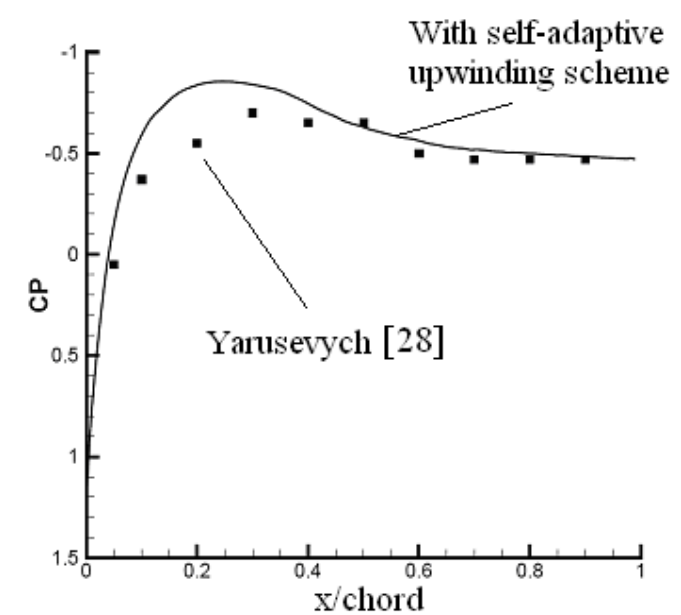

Fig. 15 Pressure coefficient over the lower surface ( $\alpha=5^{\circ}$, fine mesh).

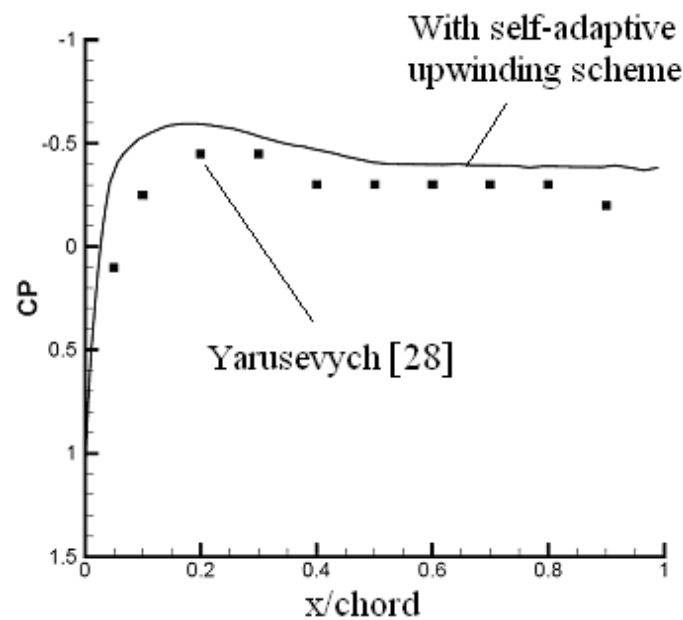

Fig. 16 Pressure coefficient over upper and lower surfaces $\left(\alpha=0^{\circ}\right.$, fine mesh).

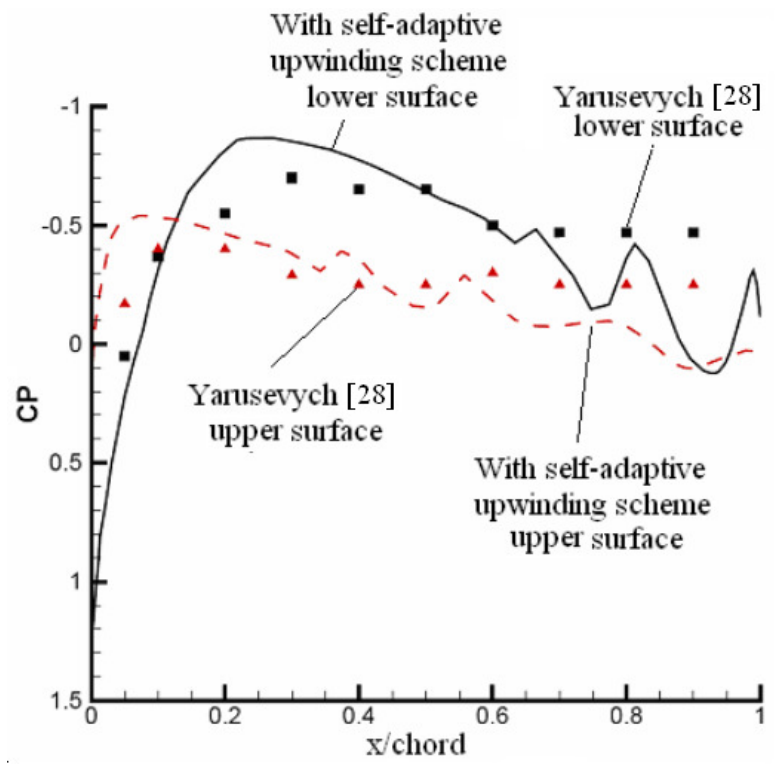

Fig. 17 Pressure coefficient over the upper and lower surfaces ( $\alpha=5^{\circ}$, coarse mesh). 


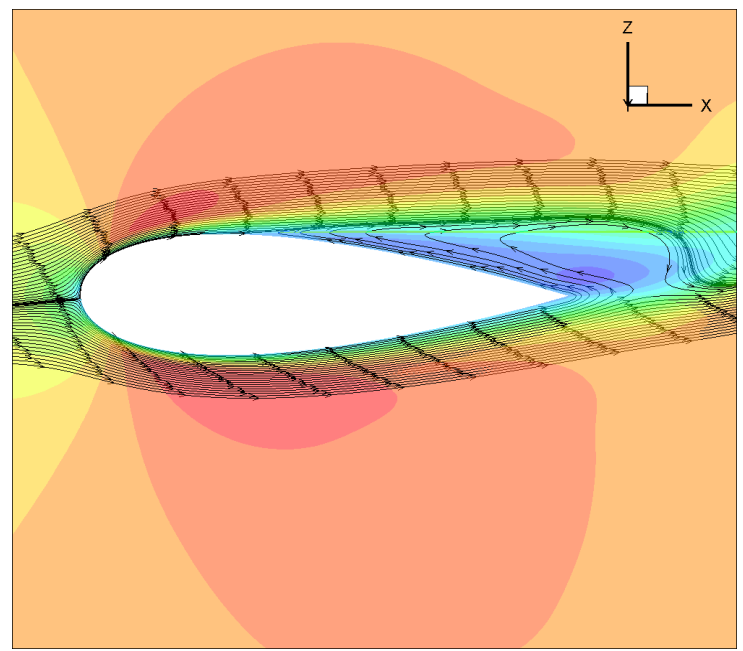

Fig. 18 Streamlines and velocity contours at time=1.0s.

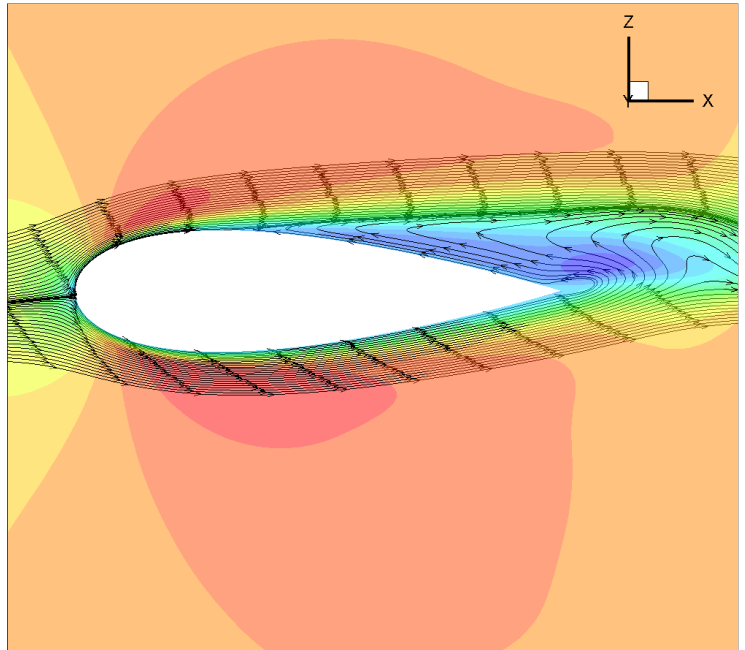

Fig. 19 Streamlines and velocity contours at time=1.015s.

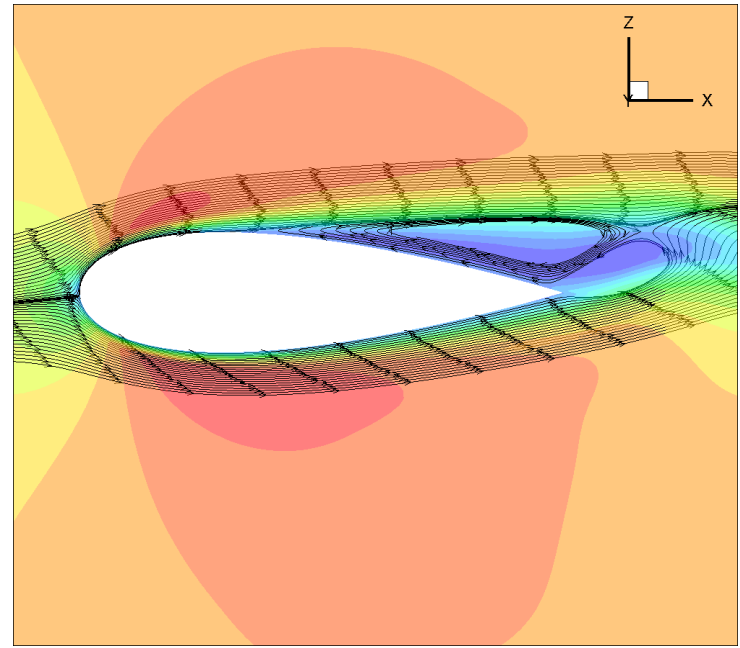

Fig. 20 Streamlines and velocity contours at time=1.033s. 


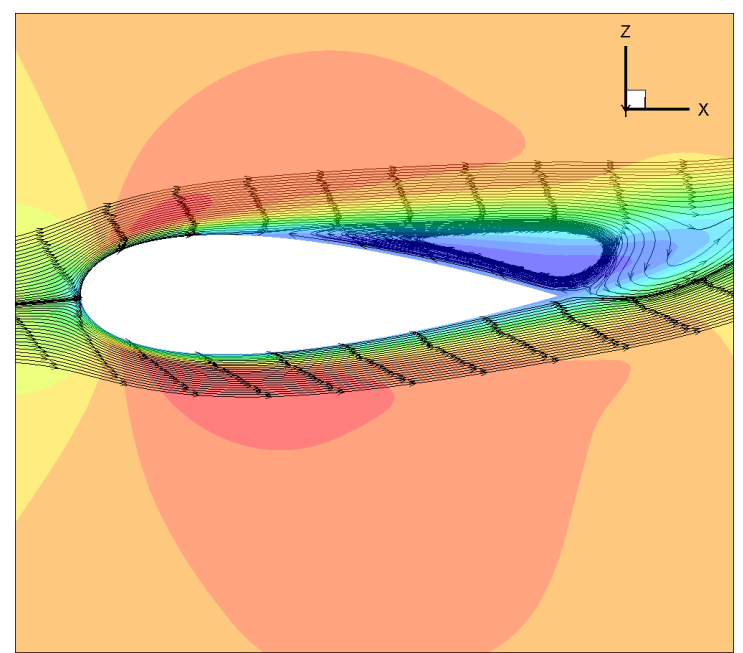

Fig. 21 Streamlines and velocity contours at time=1.049s.

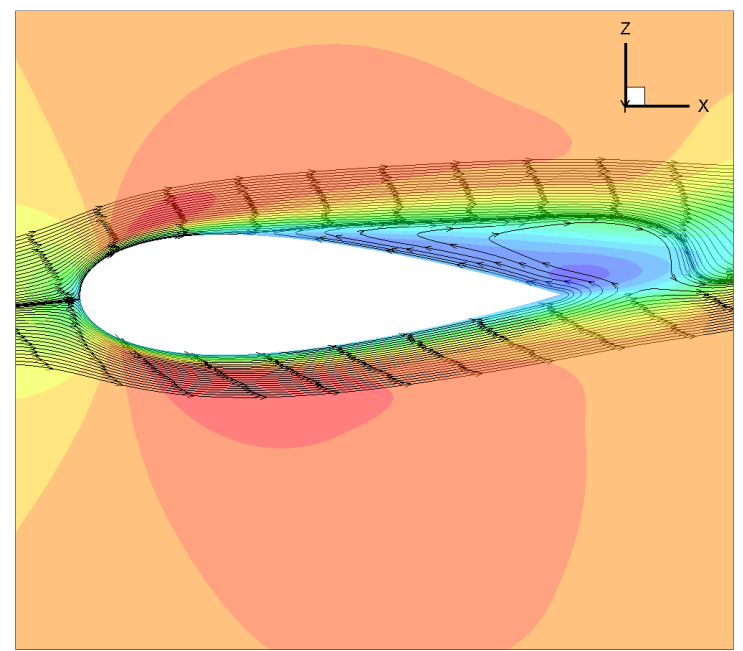

Fig. 22 Streamlines and velocity contours at time=1.067s.

As for the boundary conditions, outflow boundary condition is used because the details of the flow velocity and pressure are not known prior to the solution of the flow problem. Velocity inlet boundary condition is applied to define the flow velocity, along with all relevant scalar properties of the flow, at the inlet.

As FLUENT's user guide recommends, simulation is started by running a steady state flow simulation using a RANS turbulence model (standard $k-\varepsilon$ model) with a small Courant number. Simulation is continued until the flow field is reasonably converged. In the next step, an appropriate time step size and the other required solution parameters are set and the LES option is enabled. The simulation is continued until the flow becomes statistically steady. In this stage, the initial statistics are zeroed out and data sampling is enabled to get statistically stable data. The same mesh which was previously used for the compressible in-house code was chosen at first for the simulation 
with FLUENT; however it could not capture the separation at $\alpha=5^{\circ}$. Therefore, another mesh with finer resolution inside the boundary layer was generated $\left(\Delta \mathrm{y}^{+} \approx 0.2\right)$. For this mesh the first separation bubbles were detected at $\mathrm{x} /$ chord $=0.42$ for $\alpha=5^{\circ}$ as it is shown in Fig. 23. The surface pressure distributions are acceptable around the leading edge; but for $\mathrm{x} /$ chord larger than 0.6 , even though we expect a constant static pressure as it was predicted by the in-house code and also by the experiment; the pressure decreases on both the upper and the lower surfaces. This behavior is the result of reattachment of the flow to the surface of the wing which is not physical and therefore introduces a significant error. The different between experimental data and the fluent simulation is even more significant in Fig. 24 in which the pressure distribution on the lower surface of the wing is shown.

In conclusion, we can say that the results of the proposed scheme have been more accurate and reliable than the results of the simulation by FLUENT, despite the fact that the grid used in the later simulation was much finer than the one used for the simulation with the in-house code. As it is shown in Table 4, FLUENT's mesh is almost two times more refined in terms of the number of nodes in comparison to the mesh used by the in-house code. The surface pressure for $\alpha=0^{\circ}$ is also shown in Fig. 25. Note that in FLUEN, control volumes are the same as grid elements whereas the in-house code uses node-centered control volumes for the calculations and therefore the required degrees of freedom (D.O.F.) are 6 times more for the FLUENT in comparison to the in-house code.

Table 4 Comparison of used parameters in different numerical simulation

\begin{tabular}{ccc}
\hline \hline Numerical tool & Compressible Code & FLUENT \\
\hline Number of nodes & $3,000,000$ & $5,800,000$ \\
Number of elements & $9,500,000$ & $18,000,000$ \\
D.O.F & $5 \times 3 M$ & $5 \times 18 M$ \\
$\Delta y^{+}$ & $\approx 1$ & $\approx 0.2$ \\
No. of CPUs & 64 & 16 \\
Elapsed time & 48 hours & 45 hours \\
$C_{S}[$ Smag.] & 0.00014 & 0.00014 \\
Upwinding Parameters & & \\
\hline Upper value & 1.0 & - \\
Lower value & 0.0 & - \\
$\theta$ & -0.0001 & - \\
\hline \hline
\end{tabular}




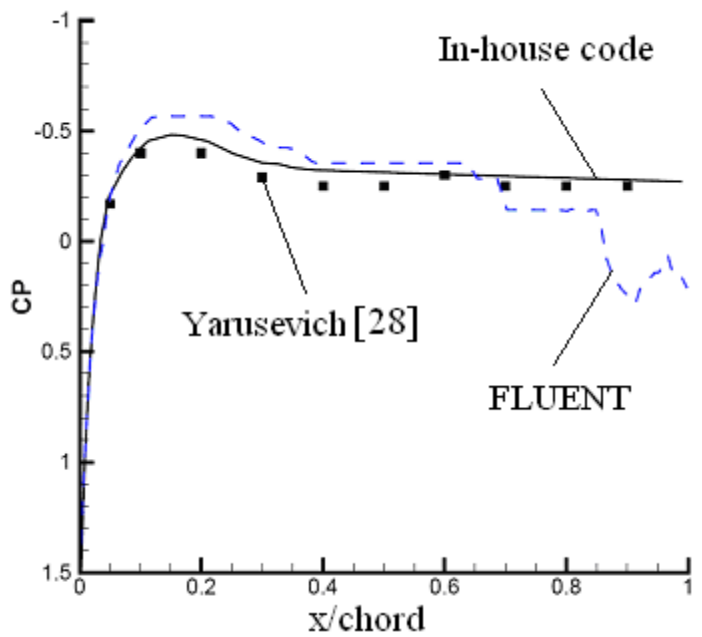

Fig. 23 Upper surface pressure distribution at $\alpha=5^{\circ}$.

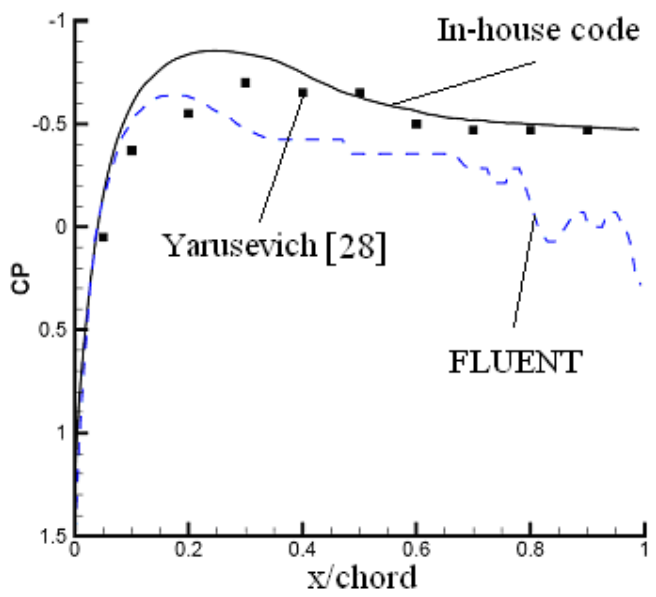

Fig. 24 Lower surface pressure distribution at $\alpha=5^{\circ}$.

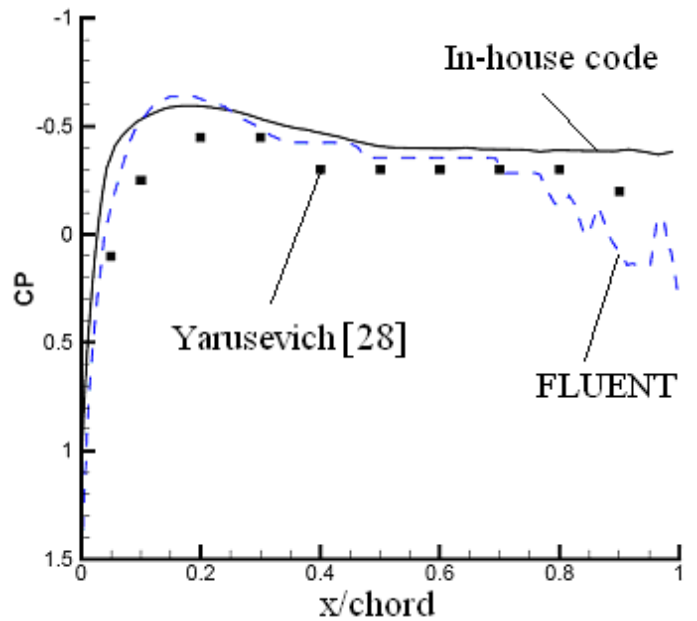

Fig.25 Surface pressure distribution at $\alpha=0^{\circ}$. 


\section{Conclusions}

A self-adaptive upwinding method for an unstructured finite-volume flow solver has been presented. The scheme tries to regulate the numerical dissipation, relying on adjustment of the upwinding term and a sensor that detects the intensity of wiggles in the flow variables. It was firstly used in a channel flow simulation to test its stability. It showed very good stability characteristic even when the noise coefficient was very high. Secondly isotropic turbulence was considered. The original scheme appeared to be over-dissipative, preventing SGS model from producing a proper LES solution. The self-adaptive upwinding method, however, improved the decaying behavior of total kinetic energy time and also the slope of energy spectrum. The wiggle detector made it possible to adjust the amount of energy in the highest modes and therefore improved the results. That adjustment, however, influenced the energy distribution on the entire spectrum and showed some undesirable effects in the lower modes. Finally, the flow separation phenomena over a NACA0025 profile at angles of attack equal to 0 and 5 degrees has been investigated. The original flux calculation scheme appeared to be over dissipative, preventing the flow to separate over the airfoil. The self-adaptive upwinding method reduced the artificial diffusion to the level of flow instability and made it possible for the separation to occur. The proposed scheme produced results which are comparable to experimental data and also more accurate and reliable than results obtained by FLUENT, despite the fact that the grid used in the later simulation was much finer than the one used for the simulation with the in-house code.

\section{References}

[1] Spyropoulos, E. T., Blaisdell, G. A., "Large-Eddy Simulation of a Spatially Evolving Supersonic Turbulent Boundary Layer Flow,” AIAA Journal, Vol. 36, 1998, pp. 1983-1990.

[2] Mary, I., Sagaut, P., "Large Eddy Simulation of Flow Around an Airfoil Near Stall," AIAA Journal, Vol. 40, 2002, pp. 1139-1145.

[3] Andersson, N., Eriksson, L. E., Davidson, L. "Large-Eddy Simulation of a Subsonic Turbulent Jets and Their Radiated Sound," AIAA Journal, Vol. 43, 2005, pp. 1899-1912.

[4] Hahn, M., Drikakis, D., "Large Eddy Simulation of Compressible Turbulence Using High-Resolution Methods," International Journal for Numerical Methods in Fluids, Vol. 47, 2005, pp. 971-977. 
[5] Carpentier, R., "Comparaison Entre des Schémas 2D de Type Roe Sur Maillages Régulier Triangule ou Quadrangle, II: Calcul au Sommet-le $\beta \gamma$ Schéma," Institut National de Recherche en Informatique et en Automatique (INRIA), Rept. 3360, France, Feb. 1980.

[6] Bui, T. T., "A Parallel, Finite-Volume Algorithm for Large Eddy Simulation of Turbulent Flows," Dryden Flight Research Center, California, NASA TM-206570, Jan. 1999.

[7] Camarri, S., Salvetti, M.V., "Large-Eddy Simulation of a Bluff-Body Flow on Unstructured Grids," International Journal for Numerical Methods in Fluids, Vol. 40, 2002, pp. 1431-1460.

[8] Ciardi, M., Saugaut, P., Klein, M., Dawes, W.N., “A Dynamic Finite Volume Scheme for Large-Eddy Simulation on Unstructured Grids,” J. of Comp. Physics, Vol. 210, 2005, pp. 632-655.

[9] Ferziger, J. H., "Direct and Large Eddy Simulation of Turbulence," CRM Proceedings and Lecture Notes, Centre de Recherches Mathématiques, Université de Montréal, Montréal, Québec, Canada, Vol. 16, 1998.

[10] Camarri, S., Salvetti, M. V., Koobus, B., “A Low-Diffusion MUSCL Scheme for LES on Unstructured Grids,” Journal of Computers and Fluids, Vol. 33, 2004, pp.1101-1129.

[11] Dahlström, S., Davidson, L., "Large Eddy Simulation Applied to a High Reynolds flow Around an Airfoil Close to Stall," $41^{\text {st }}$ AIAA Aerospace Sciences Meeting and Exhibit, Chalmers University of Technology, Sweden, 2003.

[12] Hirsch, C., "Numerical Computation on Internal and External Flows," John Wiley \& Sons, New York, 1988, Vol. 1.

[13] Cadiou, A., "NadiaLES: Theoretical Manual," Centre National De La Recherche Scientifique (CNRS), Rept. LMFA-UMR CNRS 5509, France, 2003.

[14] Larcheveque, L., Sagaut, P., Mary, I., Labbé, O., "Large-Eddy Simulation of a Compressible Flow Past a Deep Cavity," Physics of Fluids, Vol. 15, 2003, pp.193-210.

[15] Quéméré, P., Sagaut, P., "Zonal Multi-Domain RANS/LES Simulations of Turbulent Flows," International Journal for Numerical Methods in Fluids, Vol. 40, 2002, pp. 903-925.

[16] Hallo, L., Le, C., Buffet, Ribault and M., "An Implicit Mixed Finite-Element-Finite-Volume Method for Solving 3-D Turbulent Compressible Flows," International Journal for Numerical Method in Fluids, Vol. 25, 1997, pp.1241-1261. 
[17] John, V., "Large Eddy Simulation of Turbulent Incompressible Flows," Springer-Verlag Berlin Heidelberg, Germany, 2004.

[18] Knight, D., Zhou, G., Okong'o, N., Shukla, V., "Compressible Large Eddy Simulation Using Unstructured Grids," $36^{\text {th }}$ Aerospace Sciences Meeting and Exhibit, Rutgers Univ., Piscataway, NJ, Jan. 1998.

[19] Passot, T., Pouquet, A., "Numerical Simulation of Compressible Homogeneous Flows in the Turbulent Regime," J. Fluid Mechanics, Vol.181, 1987, pp. 441-466.

[20] Uddin, A., Kato, C., Yamade, Y., "Large Eddy Simulation of Homogeneous Isotropic Turbulent Flow Using the Finite Element Method," JSME, Vol. 49, 2006, pp. 102-114.

[21] Spyropoulos, E. T., Blaisdell, G. A., "Evaluation of the Dynamic Subgrid-Scale Model for Large Eddy Simulations of Compressible Turbulent Flows," $33^{\text {rd }}$ Aerospace Sciences meeting and Exhibit, Purdue University, West Lafayette, Indiana, USA, Jan. 9-12, 1995.

[22] Jimenez, J., Wray, A. A., "The Structure of Intense Vorticity in Isotropic Turbulence," J. Fluid Mechanics, Vol. 225, 1993, pp. 65-90.

[23] Hickel, S., Adams, N. A., Domaradzki, J. A., “An Adaptive Local Deconvolution Method for Implicit LES,” J. of Computational physics, Vol. 213, 2006, pp. 413-436.

[24] Comte-Bellot, G., Corrsin, S., "Simple Eulerian Time Correlations of Full and Narrow Band Velocity Signals in Grid Generated Isotropic Turbulence,” J. Fluid Mech., Vol. 48, Jul. 1971, pp. 273-337.

[25] Erlebacher, G., hussaini, M.Y., Speziale, C.G., Zang, T.A., "Toward the Large-Eddy Simulation of Compressible Turbulent Flows," J. Fluid Mech., Vol. 238, 1992, pp. 155-185.

[26] McMillan, O. J., Ferzinger, J. H., "Direct Testing of Subgrid-Scale Models,” AIAA Journal, Vol. 17, 1979, pp. $1340-1346$.

[27] Garnier, E., Mossi, M., Sagaut, P., "On the Use of Shock-Capturing Schemes for Large Eddy Simulation," Journal of Computational Physics, Vol. 153, 1999, pp. 273-311.

[28] Yarusevych, S., "Investigation of airfoil boundary layer turbulent wake development at low Reynolds numbers," Ph.D. Dissertation, University of Toronto, Toronto, Ontario, Canada, 2006.

[29] Yarusevych, S., Sullivan, P. E., and Kawall, J. G., "Coherent structures in an airfoil boundary layer and wake at low Reynolds numbers," Journal of Physics of Fluids, vol.18, issue 4, instability and transition, 2006. 
[30] Piomelli, U., and Chasnov, J. R., "Large-Eddy Simulation: Theory and Application in Transition and Turbulence Modeling,” Dordrecht, Kluwer Academic Publishers, 1996, pp. 269-336.

[31] Roberts, K. S., and Yaras, M. I., "Large-Eddy Simulation of Transition in a Separation Bubble," ASME, Vol. 128, 2006, pp. 232-238. 